\title{
STRUCTURE OF HECKE ALGEBRAS OF MODULAR FORMS MODULO $p$
}

\author{
SHAUNAK V. DEO
}

\begin{abstract}
Generalizing the recent results of Bellaïche and Khare for the level 1 case, we study the structure of the local components of the shallow Hecke algebras (i.e. Hecke algebras without $U_{p}$ and $U_{\ell}$ for all primes $\ell$ dividing the level $N$ ) acting on the space of modular forms modulo $p$ for $\Gamma_{0}(N)$ and $\Gamma_{1}(N)$. We relate them to pseudo-deformation rings and prove that in many cases, the local components are regular complete local algebras of dimension 2 .
\end{abstract}

\section{INTRODUCTION}

The $p$-adic Hecke algebra acting on modular forms of level $N$ of all weights, which is generated by the Hecke operators away from $N p$, has been well studied in the past due to its connection with $p$-adic families of modular forms and deformation rings of Galois representations (see [17] for precise definition and more details). One can similarly define a mod $p$ Hecke algebra acting on modular forms modulo $p$ of level $N$ (in the sense of Serre and Swinnerton-Dyer) of all weights. The main aim of this paper is to study the structure of these Hecke algebras acting on modular forms modulo $p$ and their relation with suitable deformation rings in characteristic $p$. These objects were previously studied by Jochnowitz ([22]), Khare ([24]), Nicolas-Serre ([27],[28]), and Bellaïche-Khare ([8]). In this article, we generalize the results of Bellaïche and Khare proved in [8] for $p \geq 5$ and $N=1$ with minor changes.

Before proceeding further, we fix some notations first. In all of this paper, we fix a prime number $p>3$ and a positive integer $N \geq 1$ not divisible by $p$. We shall denote by $K$ a finite extension of $\mathbb{Q}_{p}$, by $\mathcal{O}$ the ring of integers of $K$, by $\mathfrak{p}$ the maximal ideal of $\mathcal{O}$, by $\pi$ the generator of $\mathfrak{p}$ and by $\mathbb{F}$ the finite residue field of $\mathcal{O}$. We call $G_{\mathbb{Q}, N p}$ the Galois group of a maximal algebraic extension of $\mathbb{Q}$ unramified outside $\{\ell$ s.t. $\ell \mid N p\} \cup\{\infty\}$ over $\mathbb{Q}$. For a prime $q$ not dividing $N p$, we denote by $\operatorname{Frob}_{q} \in G_{\mathbb{Q}, N p}$ a Frobenius element at q. We denote by $c$ a complex conjugation in $G_{\mathbb{Q}, N p}$. We write $G_{\mathbb{Q}_{\ell}}$ for $\operatorname{Gal}\left(\overline{\mathbb{Q}_{\ell}} / \mathbb{Q}_{\ell}\right)$ for every prime $\ell$ dividing $N p$. There are natural maps $i_{\ell}: G_{\mathbb{Q}_{\ell}} \rightarrow G_{\mathbb{Q}, N p}$ well defined up to conjugacy. For every prime $\ell$ dividing $N p$, we write $I_{\ell}$ for the inertia subgroup of $G_{\mathbb{Q}_{\ell}}$. For a representation $\rho$ of $G_{\mathbb{Q}, N p}$, we shall denote by $\left.\rho\right|_{G_{\mathbb{Q}_{\ell}}}$ the composition of $i_{\ell}$ with $\rho$ :

2010 Mathematics Subject Classification. 11F25; 11F33; 11F80(primary).

Key words and phrases. Modular forms modulo $p$; Hecke algebras; deformation of Galois representations. 
this is a representation of $G_{\mathbb{Q}_{\ell}}$, well defined up to an isomorphism. We denote by $\omega_{p}$ : $G_{\mathbb{Q}, N p} \rightarrow \mathbb{F}^{*}$ the cyclotomic character modulo $p$.

We will now define the Hecke algebra that we want to study and the space of modular forms on which it acts. For the rest of the introduction, $\Gamma$ means either $\Gamma_{1}(N)$ or $\Gamma_{0}(N)$ and $\Gamma(M)$ means either $\Gamma_{1}(M)$ or $\Gamma_{0}(M)$ accordingly. Following [8], we shall denote by $S_{k}^{\Gamma}(\mathcal{O})$ the module of cuspidal modular forms of weight $k$ for $\Gamma$ with Fourier coefficients in $\mathcal{O}$. We see it as a submodule of $\mathcal{O}[[q]]$ by the $q$-expansion. We denote by $S_{\leq k}^{\Gamma}(\mathcal{O})$ the submodule $\sum_{i=0}^{i=k} S_{i}^{\Gamma}(\mathcal{O})$ of $\mathcal{O}[[q]]$. Note that, this sum is direct (see section 1.2 of [8]). We denote by $S_{\leq k}^{\Gamma}(\mathbb{F})$ the image of $S_{\leq k}^{\Gamma}(\mathcal{O})$ under the reduction map $\mathcal{O}[[q]] \rightarrow \mathbb{F}[[q]]$, which reduces each coefficient of a power series in $\mathcal{O}[[q]]$ modulo $\mathfrak{p}$. Thus, $S_{\leq k}^{\Gamma}(\mathbb{F})=\sum_{i=0}^{i=k} S_{i}^{\Gamma}(\mathbb{F})$, where $S_{i}^{\Gamma}(\mathbb{F})$ is the space of cuspidal modular forms of weight $i$ for $\Gamma$ over $\mathbb{F}$ in the sense of Serre and Swinnerton-Dyer i.e. it is the image of $S_{i}^{\Gamma}(\mathcal{O})$ under the reduction map considered above. The map $S_{\leq k}^{\Gamma}(\mathcal{O}) / \mathfrak{p} S_{\leq k}^{\Gamma}(\mathcal{O}) \rightarrow S_{\leq k}^{\Gamma}(\mathbb{F})$ is surjective but not an isomorphism in general. Let $S^{\Gamma}(\mathcal{O})=\cup_{k=0}^{\infty} S_{\leq k}^{\Gamma}(\mathcal{O})$ and $S^{\Gamma}(\mathbb{F})=\cup_{k=0}^{\infty} S_{\leq k}^{\Gamma}(\mathbb{F})$.

All the modules considered above have a natural action of the Hecke operators $T_{n}$ for $(n, N p)=1$. We denote by $\mathbb{T}_{k}^{\Gamma}$ the $\mathcal{O}$-subalgebra of $\operatorname{End}_{\mathcal{O}}\left(S_{<k}^{\Gamma}(\mathcal{O})\right)$ generated by the $T_{n}$ 's with $(n, N p)=1$. We denote by $A_{k}^{\Gamma}$ the $\mathbb{F}$-subalgebra of $\operatorname{End}_{\mathbb{F}}\left(S_{\leq k}^{\Gamma}(\mathbb{F})\right)$ generated by the $T_{n}$ 's with $(n, N p)=1$. From the relations between the Hecke operators, we see that $\mathbb{T}_{k}^{\Gamma}$ or $A_{k}^{\Gamma}$ is generated by the Hecke operators $T_{q}$ and $S_{q}$ for primes $q$ not dividing $N p$ (see section 1.2 of [8] for more details). Here, $S_{q}$ is the operator acting on forms of weight $k$ as the multiplication by $\langle q\rangle q^{k-2}$ where $\langle q\rangle$ is the diamond operator corresponding to $q$.

We have a natural morphism of $\mathbb{F}$-algebras $\mathbb{T}_{k}^{\Gamma} / \mathfrak{p} \mathbb{T}_{k}^{\Gamma} \rightarrow A_{k}^{\Gamma}$ which is surjective, but in general not an isomorphism. We set:

$$
\mathbb{T}^{\Gamma}=\lim _{\longleftarrow} \mathbb{T}_{k}^{\Gamma}, A^{\Gamma}=\lim _{\longleftarrow} A_{k}^{\Gamma}
$$

Thus, the Hecke algebras $\mathbb{T}^{\Gamma}$ and $A^{\Gamma}$ act on $S^{\Gamma}(\mathcal{O})$ and $S^{\Gamma}(\mathbb{F})$, respectively. We obtain a surjective map $\mathbb{T}^{\Gamma} / \mathfrak{p} \mathbb{T}^{\Gamma} \rightarrow A^{\Gamma}$ from the surjective maps considered above. We call $A^{\Gamma}$ the Hecke algebra modulo $p$ of level $\Gamma$ and this is the central object of our study.

The rings $\mathbb{T}^{\Gamma}$ and $A^{\Gamma}$ are complete and semi-local. Actually, if $\mathbb{F}$ is large enough, then, by the existence of Galois representations attached to eigenforms and by the Deligne-Serre lifting lemma, the maximal ideals and hence, the local components of both $\mathbb{T}^{\Gamma}$ and $A^{\Gamma}$ are in bijection with the set of isomorphism classes of $\Gamma$-modular Galois representations $\bar{\rho}: G_{\mathbb{Q}, N p} \rightarrow G L_{2}(\mathbb{F})$ (see section 1.2 of [8] for more details). Here and below, $\Gamma$ modular means that $\bar{\rho}$ is the semi-simplified reduction of a stable lattice for the Galois representation $\rho: G_{\mathbb{Q}, N p} \rightarrow G L_{2}(K)$ attached by Deligne to an eigenform in $S^{\Gamma}(\mathcal{O})$. Observe that, since $\bar{\rho}$ is odd, if $\bar{\rho}$ is irreducible, then it is absolutely irreducible. We define $\mathbb{T}_{\bar{\rho}}^{\Gamma}$ and $A_{\bar{\rho}}^{\Gamma}$ to be the local components of $\mathbb{T}^{\Gamma}$ and $A^{\Gamma}$ corresponding to a $\Gamma$-modular 
representation $\bar{\rho}$. These rings are complete local rings. The surjective map $\mathbb{T}^{\Gamma} / \mathfrak{p} \mathbb{T}^{\Gamma} \rightarrow A^{\Gamma}$ sends $\mathbb{T}_{\bar{\rho}}^{\Gamma} / \mathfrak{p} \mathbb{T}_{\bar{\rho}}^{\Gamma}$ onto $A_{\bar{\rho}}^{\Gamma}$. As $A^{\Gamma}$ (resp. $\mathbb{T}^{\Gamma}$ ) is semi-local and an inverse limit of artinian rings, $A^{\Gamma}$ (resp. $\mathbb{T}^{\Gamma}$ ) splits into the product of its local components. Thus, it is enough to study the structure of each local component to understand the structure of $A^{\Gamma}$, once we determine the number of its local components.

The advantage of working with the local components is that one can relate them to suitable deformation rings. By gluing pseudo-representations attached to modular eigenforms of level $\Gamma$ lifting the system of eigenvalues corresponding to a $\Gamma$-modular representation $\bar{\rho}$, one gets a pseudo-representation of $G_{\mathbb{Q}, N p}$ taking values in $A_{\bar{\rho}}^{\Gamma}$ and deforming $(\operatorname{tr} \bar{\rho}, \operatorname{det} \bar{\rho})$. Let $\tilde{R}_{\bar{\rho}}^{0}$ be the universal deformation ring with constant determinant of the pseudo-representation $(\operatorname{tr} \bar{\rho}, \operatorname{det} \bar{\rho})$ in the category of local, pro-finite $\mathbb{F}$-algebras with residue field $\mathbb{F}$. The pseudo-representation obtained above induces a local, surjective morphism $\tilde{R}_{\bar{\rho}}^{0} \rightarrow A_{\bar{\rho}}^{\Gamma}$ when either $\Gamma=\Gamma_{0}(N)$ or $p \nmid \phi(N)$ and $\Gamma=\Gamma_{1}(N)$. Otherwise, it induces a local, surjective map $\tilde{R}_{\bar{\rho}}^{0} \rightarrow\left(A_{\bar{\rho}}^{\Gamma_{1}(N)}\right)^{\text {red }}$. See the next section for more details.

We define a $\Gamma$-modular representation $\bar{\rho}$ to be unobstructed if the tangent space of $\tilde{R}_{\bar{\rho}}^{0}$ has dimension 2. If $\bar{\rho}$ is irreducible and $p \nmid \phi(N)$, then $\bar{\rho}$ is unobstructed in our sense if and only if it is unobstructed in the sense of Mazur ([26], section 1.6). But our notion is weaker than Mazur's notion if $p \mid \phi(N)$. See the last section where the notion of unobstructedness is studied in detail.

We prove the following results concerning the Hecke algebra $A_{\bar{\rho}}^{\Gamma}$ and its relation with the deformation ring $\tilde{R}_{\bar{\rho}}^{0}$ :

Theorem 1. Let $\Gamma=\Gamma_{1}(N)$ or $\Gamma_{0}(N)$. If $\bar{\rho}$ is a $\Gamma$-modular representation, then both $\tilde{R}_{\bar{\rho}}^{0}$ and $A_{\bar{\rho}}^{\Gamma}$ have Krull dimension at least 2.

Theorem 2. Suppose either $\Gamma=\Gamma_{0}(N)$ or $p \nmid \phi(N)$ and $\Gamma=\Gamma_{1}(N)$. If $\bar{\rho}$ is a $\Gamma$-modular representation which is unobstructed, then the morphism $\tilde{R}_{\bar{\rho}}^{0} \rightarrow A_{\bar{\rho}}^{\Gamma}$ is an isomorphism, and $A_{\bar{\rho}}^{\Gamma}$ is isomorphic to a power series ring in two variables $\mathbb{F}[[x, y]]$. If $p \mid \phi(N)$ and $\bar{\rho}$ is an unobstructed $\Gamma_{1}(N)$-modular representation, then the morphism $\tilde{R}_{\bar{\rho}}^{0} \rightarrow\left(A_{\bar{\rho}}^{\Gamma_{1}(N)}\right)^{\text {red }}$ is an isomorphism, and $\left(A_{\bar{\rho}}^{\Gamma_{1}(N)}\right)^{\text {red }}$ is isomorphic to a power series ring in two variables $\mathbb{F}[[x, y]]$.

In the last section, we shall give some conditions under which a $\Gamma_{1}(N)$-modular representation $\bar{\rho}$ is unobstructed and the corresponding local component $A_{\bar{\rho}}^{\Gamma_{1}(N)}$ is not reduced: see Proposition 9 and Proposition 10. We shall also give some examples of non-reduced Hecke algebras: see the remarks and discussion after Proposition 10.

Remark. (1) Our approach to prove Theorem 1 and Theorem 2 does not depend on whether $\bar{\rho}$ is irreducible or not. This is mainly because the results that we use 
from deformation theory of Galois representations are available for both reducible and irreducible $\bar{\rho}$ 's. So, the proof does not become simpler when $\bar{\rho}$ is irreducible.

(2) Note that from Theorem 2 , it follows that if $\bar{\rho}$ is a $\Gamma_{0}(N)$-modular representation which is unobstructed and if $p \nmid \phi(N)$, then the natural restriction morphism $A_{\bar{\rho}}^{\Gamma_{1}(N)} \rightarrow A_{\bar{\rho}}^{\Gamma_{0}(N)}$ is an isomorphism. In fact, if $p \nmid \phi(N)$, then this happens for any $\Gamma_{0}(N)$-modular representation $\bar{\rho}$. Indeed, fixing such a $\bar{\rho}$, we see that the corresponding system of eigenvalues for the diamond operators is trivial. As $p \nmid \phi(N)$, by Hensel's lemma, any system of eigenvalues lifting $\bar{\rho}$ is trivial for the diamond operators. Hence, the diamond operators act trivially on $S^{\Gamma_{1}(N)}(\mathcal{O})_{\bar{\rho}}$. Therefore, $S^{\Gamma_{1}(N)}(\mathcal{O})_{\bar{\rho}}=S^{\Gamma_{0}(N)}(\mathcal{O})_{\bar{\rho}}$. So, $\mathbb{T}_{\bar{\rho}}^{\Gamma_{1}(N)}$ and $\mathbb{T}_{\bar{\rho}}^{\Gamma_{0}(N)}$ are isomorphic. Thus, $A_{\bar{\rho}}^{\Gamma_{1}(N)}$ and $A_{\bar{\rho}}^{\Gamma_{0}(N)}$ are isomorphic for all the $\Gamma_{0}(N)$-modular representations $\bar{\rho}$ if $p$ does not divide $\phi(N)$. However, this argument breaks down if $p$ divides $\phi(N)$.

Theorem 3. Assume that $\bar{\rho}$ is a $\Gamma_{1}(N)$-modular representation coming from a newform of level $N$ and is absolutely irreducible after restriction to the Galois group of $\mathbb{Q}\left(\zeta_{p}\right)$. If $\ell|N, p| \ell^{2}-1$ and $\left.\bar{\rho}\right|_{G_{\mathbb{Q}_{\ell}}}$ is unramified, then assume $\ell^{2} \mid N$. If $\ell|N, p| \ell-1,\left.\bar{\rho}\right|_{G_{\mathbb{Q}_{\ell}}}$ is reducible, ramified and not a sum of two ramified characters, then assume that the highest power of $\ell$ dividing $N$ is greater than the highest power of $\ell$ dividing the Artin conductor of $\bar{\rho}$. If $\left.\bar{\rho}\right|_{G_{\mathbb{Q}_{p}}}$ is reducible, assume, in addition, that $\left.\bar{\rho}\right|_{G_{\mathbb{Q}_{p}}}$ is not isomorphic to $\chi \otimes\left(\begin{array}{ll}1 & * \\ 0 & 1\end{array}\right)$ nor to $\chi \otimes\left(\begin{array}{cc}1 & * \\ 0 & \omega_{p}\end{array}\right)$, where $\chi$ is any character $G_{\mathbb{Q}_{p}} \rightarrow \mathbb{F}^{*}$. Then $A_{\bar{\rho}}^{\Gamma_{1}(N)}$ has Krull dimension 2. Moreover, $\left(\tilde{R}_{\bar{\rho}}^{0}\right)^{\text {red }}$ is isomorphic to $\left(A_{\bar{\rho}}^{\Gamma_{1}(N)}\right)^{\text {red }}$.

Similarly, if $\bar{\rho}$ is a $\Gamma_{0}(N)$-modular representation satisfying the hypotheses as above, then $A_{\bar{\rho}}^{\Gamma_{0}(N)}$ has Krull dimension 2. Moreover, if $p$ does not divide $\phi(N)$, then $\left(\tilde{R}_{\bar{\rho}}^{0}\right)^{\text {red }}$ is isomorphic to $\left(A_{\bar{\rho}}^{\Gamma_{0}(N)}\right)^{r e d}$.

These results are generalizations of Theorem III, Theorem I and Theorem II of [8], respectively. Note that, it is possible to have $\bmod p$ deformation ring with constant determinant which are non-reduced but we do not know of any such examples.

Remark. If $p \nmid N$, then we know that the module of $\bmod p$ modular forms for $\Gamma_{1}(N)$ is the same as the module of $\bmod p$ modular forms for $\Gamma_{1}\left(N p^{e}\right)$ for $e \geq 1$ (See the remark after corollary I.3.6 on page 23 of [19] for more details). Hence, the corresponding mod $p$ Hecke algebras are also the same. So, even though we are assuming that $p \nmid N$, all the theorems that we prove for mod $p$ Hecke algebras for $\Gamma_{1}(N)$ above will still be true without this assumption on $N$.

Note that, Theorem 2 easily follows from Theorem 1 and the definition of unobstructed. 
The idea of the proof of Theorem 1 is similar to the idea used in proving Theorem III in [8]. So, we try to find a relation between the characteristic 0 Hecke algebra $\mathbb{T}_{\bar{\rho}}^{\Gamma}$ with the characteristic $p$ Hecke algebra $A_{\bar{\rho}}^{\Gamma}$. However, there are some difficulties, such as $S^{\Gamma}(\mathcal{O}) \otimes_{\mathcal{O}} \mathbb{F}$ not being isomorphic to $S^{\Gamma}(\mathbb{F})$, in comparing them directly. To overcome this problem, like [8], we also work with the divided congruence modules of Katz. Using the results of Katz and the methods of [8], we get the relation between characteristic 0 and characteristic $p$ full Hecke algebras i.e. the relation between the characteristic 0 and characteristic $p$ Hecke algebras generated by the Hecke operators $T_{q}, q S_{q}$ for primes $q$ not dividing $N p, U_{\ell}$ for primes $\ell$ dividing $N$ and $U_{p}$.

Now, we need to analyze how the addition of the operators $U_{\ell}$ 's and $U_{p}$ changes our Hecke algebras in characteristic 0 and $p$. We can control the change caused by the $U_{p}$ operator in a similar way as is done in the level 1 case in [8]. This allows us to get a relation between Hecke algebras in characteristic 0 and $p$ generated by Hecke operators away from $p$. Note that, the proof for $N=1$ case gets over at this step. However, for $N>1$, we still need to study the effect of adding the extra operators $U_{\ell}$ 's for primes $\ell$ dividing $N$ to our original Hecke algebras. This differentiates the case of $N>1$ from $N=1$. In this direction, we prove that in characteristic 0 , if $\bar{\rho}$ is new i.e. if $\bar{\rho}$ is not $\Gamma\left(N^{\prime}\right)$-modular for any proper divisor $N^{\prime}$ of $N$, then the operator $U_{\ell}$ acting on $S^{\Gamma}(\mathcal{O})_{\bar{\rho}}$ is integral over $\mathbb{T}_{\bar{\rho}}^{\Gamma}$ for every prime $\ell$ dividing $N$. This gives us the finiteness of the $U_{\ell}$ 's over the mod $p$ Hecke algebra automatically for a new $\bar{\rho}$ and, along with the Gouvêa-Mazur infinite fern argument, leads us to Theorem 1 in those cases. Finally, we prove that if $\bar{\rho}$ is also a $\Gamma(M)$-modular representation for some $M$ dividing $N$, then the natural map $A_{\bar{\rho}}^{\Gamma} \rightarrow A_{\bar{\rho}}^{\Gamma(M)}$ is surjective using the pseudo-representation attached to it and the Chebotarev density theorem. Theorem 1 in the case where $\bar{\rho}$ is new, along with the surjectivity established above, leads us to Theorem 1 for all the local components of the mod $p$ Hecke algebra. We would like to point out that, in contrast with [8], our method does not give the precise kernel of the map $\mathbb{T}_{\bar{\rho}}^{\Gamma} \rightarrow A_{\bar{\rho}}^{\Gamma}$ in all the cases while proving Theorem 1 . However, in many cases, we can find the kernel up to some nilpotence.

To prove Theorem 3, we need to use a result of Böckle and flatness of $\mathbb{T}_{\bar{\rho}}^{\Gamma_{1}(N)}$ over the Iwasawa algebra $\mathcal{O}[[T]]$ which is proved in the same way as in [8]. This, along with Theorem 1, would imply the first part of Theorem 3. We prove, using techniques and results similar to the ones sketched in the previous paragraph, that the map $\mathbb{T}_{\bar{\rho}}^{\Gamma_{1}(N)} /(\mathfrak{p}, T) \rightarrow A_{\bar{\rho}}^{\Gamma_{1}(N)}$ has nilpotent kernel if $\bar{\rho}$ satisfies the hypotheses of Theorem 3. We use it, along with the results of Böckle, to conclude the second part of Theorem 3 for the $\Gamma_{1}(N)$ case. The theorem for the $\Gamma_{0}(N)$ case then follows easily from the theorem for the $\Gamma_{1}(N)$ case. 
Finally, we would like to remark on possible generalization of these results to the case of Hilbert modular forms. A well behaved theory for $\bmod p$ Hilbert modular forms with arbitrary weights, which includes a lot of tools and facts for $\bmod p$ modular forms that we use, is available (see the works of Andreatta-Goren ([1]) for more details). Moreover, an analogue of Gouvêa-Mazur infinite fern argument is also true for certain local components of the $p$-adic Hecke algebra acting on the space of Hilbert modular forms due to work of Chenevier (see Theorem 5.9 of [13]). However, contrary to the case of modular forms, the theory of divided congruence modules of Katz and properties of Hecke operators acting on them is not known for Hilbert modular forms. We expect results similar to what we have proved above to hold for Hilbert modular forms once we know the theory of divided congruence modules of Hilbert modular forms and the infinite fern argument for all the local components. The results will depend on the space of $\bmod p$ Hilbert modular forms we consider. For instance, we expect the lower bound on the Krull dimension of the mod $p$ Hecke algebra for Hilbert modular forms of parallel weights to be 2, while the corresponding lower bound for the mod $p$ Hecke algebra for Hilbert modular forms of arbitrary weights to be $2 n$, where $n$ is the degree of extension of the totally real field over $\mathbb{Q}$.

Acknowledgements: I would like to thank my Ph.D. advisor Joël Bellaïche for suggesting this problem to me and for his patience, guidance, encouragement and support during the completion of this paper. His suggestions have also played an instrumental role in making the exposition clearer and simplifying a lot of proofs. I would also like to thank Aditya Karnataki for numerous helpful mathematical discussions. I would like to thank the referee as well for a careful reading and helpful suggestions.

\section{Deformation Rings And Hecke Algebras}

The goal of this section is to relate mod $p$ Hecke algebras with appropriate deformation rings.

Using [3, Step 1 of the proof of Theorem 1], which is essentially an argument of gluing pseudo-representations attached to modular eigenforms of a fixed level and all weights, we get the following lemma (see [8, Proposition 2] also):

Lemma 1. Let $\Gamma$ be either $\Gamma_{0}(N)$ or $\Gamma_{1}(N)$. For a $\Gamma$-modular representation $\bar{\rho}$, there exists a unique continuous pseudo-representation $\left(\tau^{\Gamma}, \delta^{\Gamma}\right): G_{\mathbb{Q}, N p} \rightarrow \mathbb{T}_{\bar{\rho}}^{\Gamma}$ such that $\tau^{\Gamma}(c)=$ $0, \tau^{\Gamma}\left(\right.$ Frob $\left._{q}\right)=T_{q}$ and $\delta^{\Gamma}\left(\right.$ Frob $\left._{q}\right)=q S_{q}$ for all the primes $q$ not dividing $N p$. We have $\tau^{\Gamma}\left(\bmod m_{\mathbb{T} \bar{\rho}}\right)=\operatorname{tr} \bar{\rho}, \delta^{\Gamma}\left(\bmod m_{\mathbb{T}_{\bar{\rho}}^{\Gamma}}\right)=\operatorname{det} \bar{\rho} . \quad$ By composing $\left(\tau^{\Gamma}, \delta^{\Gamma}\right)$ with the natural morphism $\mathbb{T}_{\bar{\rho}}^{\Gamma} \rightarrow A_{\bar{\rho}}^{\Gamma}$, we get a pseudo-representation $\left(\tilde{\tau}^{\Gamma}, \tilde{\delta}^{\Gamma}\right): G_{\mathbb{Q}, N p} \rightarrow A_{\bar{\rho}}^{\Gamma}$ lifting $(\operatorname{tr} \bar{\rho}, \operatorname{det} \bar{\rho})$. 
A deformation $(t, d)$ of a pseudo-representation $(\bar{t}, \bar{d})$ is called a deformation with constant determinant if $d=\bar{d}$.

Lemma 2. If $\Gamma=\Gamma_{0}(N)$ or if $p \nmid \phi(N)$ and $\Gamma=\Gamma_{1}(N)$, then for a $\Gamma$-modular representation $\bar{\rho},\left(\tilde{\tau}^{\Gamma}, \tilde{\delta}^{\Gamma}\right)$ is a deformation of $(\operatorname{tr} \bar{\rho}, \operatorname{det} \bar{\rho})$ with constant determinant.

Proof. Let $q$ be a prime not dividing $N p$. Note that, in the $\Gamma_{0}(N)$ case, $S_{q}$ acts as multiplication by $q^{k-2}$ on a weight $k$ modular form with Fourier coefficients in $\mathcal{O}$. If two modular forms of level $N$ are congruent modulo $\mathfrak{p}$, then their weights are congruent modulo $p-1$. So, $S_{q}$ acts like a constant on $S^{\Gamma_{0}(N)}(\mathbb{F})_{\bar{\rho}}$ for every $\bar{\rho}$. Since $\tilde{\delta}^{\Gamma}\left(\operatorname{Frob}_{q}\right)=q S_{q}$ and the set of $\operatorname{Frob}_{q}$ for primes $q$ not dividing $N p$ is dense in $G_{\mathbb{Q}, N p}$, we get that $\tilde{\delta}$ is constant and we are done in the $\Gamma_{0}(N)$ case.

Applying the same reasoning to the $\Gamma_{1}(N)$ case, we get that, for every prime $q$ not dividing $N p, S_{q}$ acts like $c_{q}\langle q\rangle$ on $S^{\Gamma_{1}(N)}(\mathbb{F})_{\bar{\rho}}$ where $c_{q}$ is an invertible constant. Since $S_{q} \in A_{\bar{\rho}}^{\Gamma_{1}(N)}$, it follows that $\langle q\rangle \in A_{\bar{\rho}}^{\Gamma_{1}{ }^{(N)}}$ for every prime $q$ not dividing $N p$. If $p$ does not divide $\phi(N)$, then the order of every diamond operator $\langle q\rangle$ is co-prime to $p$. We have chosen $\mathbb{F}$ to be large enough so that it contains all the mod $p$ system of eigenvalues. Thus, by Hensel's lemma, $\langle q\rangle$ and hence $S_{q}$, will be constant in $A_{\bar{\rho}}^{\Gamma_{1}(N)}$ for every $\bar{\rho}$ and every prime $q$ not dividing $N p$. Therefore, $\tilde{\delta}^{\Gamma}$ is constant in the $\Gamma_{1}(N)$ case if $p$ does not divide $\phi(N)$ by the same argument as in the $\Gamma_{0}(N)$ case.

If $p \mid \phi(N)$, then the determinant $\tilde{\delta}^{\Gamma}$ may not be constant. See the last section for more details. If the order of $\langle q\rangle$ is $p^{e}$, then $(\langle q\rangle-1)^{p^{e}}=0$ in $A_{\bar{\rho}}^{\Gamma_{1}(N)}$. Therefore, in $\left(A_{\bar{\rho}}^{\Gamma_{1}(N)}\right)^{\mathrm{red}}$, we have $\langle q\rangle=1$ for all such $\langle q\rangle$ 's. Thus, from the proof of the lemma above, it follows that if $p \mid \phi(N)$, then the determinant $\left(\tilde{\delta}^{\Gamma_{1}(N)}\right)^{\text {red }}: G_{\mathbb{Q}, N p} \rightarrow\left(\left(A_{\bar{\rho}}^{\Gamma_{1}(N)}\right)^{\text {red }}\right)^{*}$ is constant.

Let $R_{\bar{\rho}}$ be the universal deformation ring of the pseudo-representation $(\operatorname{tr} \bar{\rho}, \operatorname{det} \bar{\rho})$ in the category of local pro-finite $\mathcal{O}$-algebras with residue field $\mathbb{F}, \tilde{R}_{\bar{\rho}}$ be the corresponding universal deformation ring $\bmod \mathfrak{p}$ and $\tilde{R}_{\bar{\rho}}^{0}$ be the corresponding universal deformation ring $\bmod \mathfrak{p}$ with constant determinant (see [12], [8, Section 1.4] for more details regarding the existence and properties of these rings). For a $\Gamma$-modular representation $\bar{\rho}$, the pseudorepresentation $\left(\tau^{\Gamma}, \delta^{\Gamma}\right)$ defines a local morphism $R_{\bar{\rho}} \rightarrow \mathbb{T}_{\bar{\rho}}^{\Gamma}$ which is identity modulo their maximal ideals. Similarly, we get a local morphism $\tilde{R}_{\bar{\rho}} \rightarrow A_{\bar{\rho}}^{\Gamma}$. From the previous paragraphs, we see that the morphism $\tilde{R}_{\bar{\rho}} \rightarrow A_{\bar{\rho}}^{\Gamma}$ factors through $\tilde{R}_{\bar{\rho}}^{0}$ if $\Gamma=\Gamma_{0}(N)$ or if $p \nmid \phi(N)$ and $\Gamma=\Gamma_{1}(N)$. However, this is not true in general. But, from above, we see that for a $\Gamma_{1}(N)$-modular representation $\bar{\rho}$, the map $\tilde{R}_{\bar{\rho}} \rightarrow\left(A_{\bar{\rho}}^{\Gamma_{1}(N)}\right)^{\text {red }}$ factors through $\tilde{R}_{\bar{\rho}}^{0}$. All the morphisms considered above are surjective. Indeed, for a prime $q$ not dividing $N p$, the images of the trace and the determinant, coming from the universal pseudo-representation, of $\operatorname{Frob}_{q}$ under the morphisms above are $T_{q}$ and $q S_{q}$, respectively. 


\section{Relation between the full Hecke algebras in characteristic 0 and $p$}

The goal of this section is to get a relation between the full Hecke algebras like the one obtained in Proposition 16 in [8]. We will be following the approach of [8] to get a result similar to Proposition 16 of [8] for both the cases, $\Gamma_{1}(N)$ and $\Gamma_{0}(N)$. We will briefly recall, without proofs, some important results of the theory of divided congruences of Katz needed for our purpose. Then, we will state some results about the comparisons of various Hecke algebras which are similar to the results given in sections 3, 4, 5 and 6 of [8]. Since the proofs of these results are more or less the same as the ones given in [8], we shall not give them in full detail here. Instead, we will mostly refer readers to the proofs of corresponding results in [8] and will provide some additional details when required. In this section, we will follow the notation of [8] for the divided congruence modules and the Hecke algebras along with an additional index representing the level. For instance, $D^{\Gamma_{1}(N)}(\mathcal{O})$ will represent the divided congruence module of cuspidal forms for $\Gamma_{1}(N)$ over $\mathcal{O}$. These modules and the Hecke algebras acting on them are defined in exactly the same way as their level 1 counterparts in [8] after making appropriate level changes. Main references for this section are [23], [21] and [8]. Throughout this section, $\Gamma$ means either $\Gamma_{1}(N)$ or $\Gamma_{0}(N)$.

3.1. The divided congruence modules of Katz. In this and the following subsection, we quickly list all the results that are needed from the theory of divided congruence modules. These results are the level $N$ counterparts of the results that appear in [8].

We will now define the divided congruence modules of Katz. Let $S_{\leq k}^{\Gamma}(K)$ be the subspace of $K[[q]]$ given by $\sum_{i=0}^{i=k} S_{i}^{\Gamma}(K)$, where $S_{i}^{\Gamma}(K)$ is the space of cusp forms of weight $i$ and level $\Gamma$ with Fourier coefficients in $K$ which is identified as a subspace of $K[[q]]$ via $q$-expansions. Let $D_{\leq k}^{\Gamma}(\mathcal{O})$ be the $\mathcal{O}$-submodule of $\mathcal{O}[[q]]$ given by the intersection of $S_{\leq k}^{\Gamma}(K)$ with $\mathcal{O}[[q]]$. They are called divided congruence modules because they capture congruences between cusp forms of different weights (see Remark 3 of section 2 of [8] for more details). We define $D_{\leq k}^{\Gamma}(\mathbb{F})$ as the image of $D_{\leq k}^{\Gamma}(\mathcal{O})$ under the reduction map $\mathcal{O}[[q]] \rightarrow \mathbb{F}[[q]]$. Let $D^{\Gamma}(\mathcal{O})=\cup_{k=0}^{\infty} D_{\leq k}^{\Gamma}(\mathcal{O})$ and $D^{\Gamma}(\mathbb{F})=\cup_{k=0}^{\infty} D_{\leq k}^{\Gamma}(\mathbb{F})$. We call $D^{\Gamma}(\mathcal{O})$ the divided congruence module of cuspidal forms of level $\Gamma$ and $D^{\Gamma}(\mathbb{F})$ the divided congruence module of cuspidal forms modulo $p$ of level $\Gamma$. See section 2 of [8] for more details.

Lemma 3. The natural map $D_{\leq k}^{\Gamma}(\mathcal{O}) \otimes_{\mathcal{O}} \mathbb{F} \rightarrow D_{\leq k}^{\Gamma}(\mathbb{F})$ is an isomorphism.

Proof. If $f \in D_{\leq k}^{\Gamma}(\mathcal{O})$ lies in the kernel of the natural surjective map $D_{\leq k}^{\Gamma}(\mathcal{O}) \rightarrow D_{\leq k}^{\Gamma}(\mathbb{F})$, then $\frac{f}{\pi}$ lies in both $S_{\leq k}^{\Gamma}(K)$ and $\mathcal{O}[[q]]$. Hence, it lies in $D_{\leq k}^{\Gamma}(\mathcal{O})$ which implies the lemma. See the proof of Lemma 5 of [8]. 
We now recall, without proofs, two theorems of Katz which relate the divided congruence module of cuspidal forms mod $p$ of level $\Gamma$ with the space of mod $p$ cuspforms of level $\Gamma$ :

Proposition 1. (Katz)

(1) There exists a unique action of $\mathbb{Z}_{p}^{*} \times(\mathbb{Z} / N \mathbb{Z})^{*}$ on $D^{\Gamma_{1}(N)}(\mathcal{O})$ denoted by $((x, y), f)$ $\mapsto(x, y)$.f , such that for $(x, y) \in \mathbb{Z}_{p}^{*} \times(\mathbb{Z} / N \mathbb{Z})^{*}$, and $f \in S_{k}^{\Gamma_{1}(N)}(\mathcal{O}) \subset D^{\Gamma_{1}(N)}(\mathcal{O})$, $(x, y) \cdot f=x^{k}\langle y\rangle f$.

(2) There exists a unique action of $\mathbb{Z}_{p}^{*}$ on $D^{\Gamma_{0}(N)}(\mathcal{O})$ denoted by $(x, f) \mapsto x . f$ such that for $x \in \mathbb{Z}_{p}^{*}$, and $f \in S_{k}^{\Gamma_{0}(N)}(\mathcal{O}) \subset D^{\Gamma_{0}(N)}(\mathcal{O})$, x.f $=x^{k} f$

See $[23$, Cor 1.7] for the proof.

Theorem 4. (Katz) The space $S^{\Gamma}(\mathbb{F})$ is the space of invariants of $1+p \mathbb{Z}_{p}$ acting on $D^{\Gamma}(\mathbb{F})$.

This is proved in [23, Sec.4]; see also [21, Thm. 1.1].

The two results of Katz recalled in this subsection are proved only for $p>3$. We do not know whether they also hold for $p=2,3$.

3.2. Hecke operators on the divided congruence modules. We can define the Hecke operators $T_{q}, S_{q}$ for primes $q$ not dividing $N p$ and $U_{\ell}$ for primes $\ell$ dividing $N$ on $D^{\Gamma}(\mathcal{O})$. Their action on the $q$-expansions is given in the same way as it is given on the $q$-expansions of the classical modular forms.

See the proof of Corollary and Definition 7 of [8] and [21, Page 243] for more details.

Now we introduce partially full Hecke algebras which are generated by the $U_{\ell}$ 's for every prime $\ell$ dividing $N$ along with the $T_{n}$ 's for $(n, N p)=1$. We add an extra pf in the index to denote these Hecke algebras. Thus, $\mathbb{T}_{k}^{\Gamma, p f}$ is the $\mathcal{O}$-subalgebra of $\operatorname{End}_{\mathcal{O}}\left(S_{\leq k}^{\Gamma}(\mathcal{O})\right)$ generated by the $T_{n}$ 's with $(n, N p)=1$ and $U_{\ell}$ 's with $\ell$ prime dividing $N$, while $A_{k}^{\Gamma, p f}$ is the $\mathbb{F}$-subalgebra of $\operatorname{End}_{\mathbb{F}}\left(S_{\leq k}^{\Gamma}(\mathbb{F})\right)$ generated by the $T_{n}$ 's with $(n, N p)=1$ and $U_{\ell}$ 's with $\ell$ prime dividing $N$. We can consider the projective limits:

$$
\mathbb{T}^{\Gamma, \mathrm{pf}}=\lim _{\longleftarrow} \mathbb{T}_{k}^{\Gamma, \mathrm{pf}}, A^{\Gamma, \mathrm{pf}}=\lim _{\longleftarrow} A_{k}^{\Gamma, \mathrm{pf}}
$$

Lemma 4. (1) The sub-algebra of $E n d_{\mathcal{O}}\left(D_{\leq k}^{\Gamma}(\mathcal{O})\right)$ generated by the Hecke operators $T_{q}, S_{q}$ for primes $q$ not dividing $N p$ and $U_{\ell}$ for primes $\ell$ dividing $N$ is naturally isomorphic to $\mathbb{T}_{k}^{\Gamma, p f}$.

(2) The sub-algebra of $\operatorname{End}_{\mathcal{O}}\left(D_{\leq k}^{\Gamma}(\mathcal{O})\right)$ generated by the Hecke operators $T_{q}, S_{q}$ for primes $q$ not dividing $N p$ is naturally isomorphic to $\mathbb{T}_{k}^{\Gamma}$. 
Proof. Both parts of the lemma follow from the observations that $S_{\leq k}^{\Gamma}(\mathcal{O})$ is a co-torsion submodule of $D_{\leq k}^{\Gamma}(\mathcal{O})$ and the action of Hecke operators on $D_{\leq k}^{\Gamma}(\mathcal{O})$ extends their action on $S_{\leq k}^{\Gamma}(\mathcal{O})$. Indeed, as $S_{\leq k}^{\Gamma}(\mathcal{O})$ is a co-torsion submodule of $D_{\leq k}^{\Gamma}(\mathcal{O})$, if $f \in D_{\leq k}^{\Gamma}(\mathcal{O})$, then $\pi^{n} f \in S_{\leq k}^{\Gamma}(\mathcal{O})$ for some $n$ which implies that an Hecke operator vanishing on $S_{\leq k}^{\Gamma}(\mathcal{O})$ also vanishes on $D_{\leq k}^{\Gamma}(\mathcal{O})$. See the proof of Lemma 8 of [8] for more details.

Lemma 5. (1) The homomorphism $\phi: \mathbb{Z}_{p}^{*} \rightarrow \operatorname{End}_{\mathcal{O}}\left(D^{\Gamma_{1}(N)}(\mathcal{O})\right)$, defined by $\phi(x) f=$ $(x, 1)$. $f$ for $f \in D^{\Gamma_{1}(N)}(\mathcal{O})$, takes values in the sub-algebra $\mathbb{T}^{\Gamma_{1}(N)}$ and hence, in $\mathbb{T}^{\Gamma_{1}(N), p f}$.

(2) The homomorphism $\phi: \mathbb{Z}_{p}^{*} \rightarrow \operatorname{End}_{\mathcal{O}}\left(D^{\Gamma_{0}(N)}(\mathcal{O})\right)$, defined by $\phi(x) f=x$.f for $f \in$ $D^{\Gamma_{0}(N)}(\mathcal{O})$ takes values in the sub-algebra $\mathbb{T}^{\Gamma_{0}(N)}$ and hence, in $\mathbb{T}^{\Gamma_{0}(N), p f}$.

Proof. The proof of part 1 is almost the same as the proof of Lemma 9 of [8]. We only need to change the last step of the proof slightly, so we sketch it briefly here. Following the same proof, we see that $\mathbb{T}^{\Gamma_{1}(N)}$ is a closed subset of $\operatorname{End}_{\mathcal{O}}\left(D^{\Gamma_{1}(N)}(\mathcal{O})\right)$ under the weak topology, and the map $\phi: \mathbb{Z}_{p}^{*} \rightarrow \operatorname{End}_{\mathcal{O}}\left(D^{\Gamma_{1}(N)}(\mathcal{O})\right)$ is continuous for the weak topology. For a prime $q$ which does not divide $N p$ and is $1(\bmod N)$, one has $\phi(q)=q^{2} S_{q} \in \mathbb{T}^{\Gamma_{1}(N)}$, since $\langle q\rangle$ is the trivial operator as $q$ is $1(\bmod N)$. If $x \in \mathbb{Z}_{p}^{*}$, there exists, by the Chinese remainder theorem and Dirichlet's theorem on primes in arithmetic progression, a sequence of primes $q_{n}$ (different from primes dividing $N p$ ) which are $1(\bmod N)$ and which converges to $x$-adically. Hence, $\phi\left(q_{n}\right)$ converges to $\phi(x)$ in $\operatorname{End}_{\mathcal{O}}\left(D^{\Gamma_{1}(N)}(\mathcal{O})\right)$. Therefore, $\phi(x) \in \mathbb{T}^{\Gamma_{1}(N)}$. The proof of the part 2 is the same as the proof of Lemma 9 of $[8]$.

Let $\Lambda$ be the Iwasawa algebra, $\mathcal{O}\left[\left[1+p \mathbb{Z}_{p}\right]\right]$. By choosing a topological generator of $1+p \mathbb{Z}_{p}$, say $1+p$, one gets an isomorphism $\Lambda \simeq \mathcal{O}[[T]]$. Under this isomorphism, the maximal ideal $m_{\Lambda}$ of $\Lambda$ gets mapped to $(\pi, T)$. We get a morphism $\psi: \Lambda \rightarrow \mathbb{T}^{\Gamma}$ of $\mathcal{O}$ algebras from the group homomorphism $\phi: 1+p \mathbb{Z}_{p} \rightarrow\left(\mathbb{T}^{\Gamma}\right)^{*}$. Using the morphism $\psi$, we can consider $\mathbb{T}^{\Gamma}$ as a $\Lambda$-algebra.

3.3. Divided congruence modules of level $\Gamma_{0}(N p)$ and $\Gamma_{1}(N p)$. In this subsection, we consider the divided congruence modules of cuspidal forms for levels $\Gamma_{0}(N p)$ and $\Gamma_{1}(N p)$. They are defined in the same way as in the level $N$ case after just changing the level. For the rest of this section, $\Gamma$ still means $\Gamma_{0}(N)$ or $\Gamma_{1}(N)$ and $\Gamma(p)$ means either $\Gamma_{0}(N p)$ or $\Gamma_{1}(N p)$ accordingly.

Proposition 2. The closures of $D^{\Gamma}(\mathcal{O})$ and $D^{\Gamma(p)}(\mathcal{O})$ in $\mathcal{O}[[q]]$, which is provided with the topology of uniform convergence, are equal.

Proof. See [19, Proposition I.3.9] and section 1 of [21]. 
Corollary 1. There is an isomorphism preserving q-expansions of $D^{\Gamma(p)}(\mathcal{O}) \otimes_{\mathcal{O}} \mathbb{F} \simeq$ $D^{\Gamma}(\mathbb{F})$.

Corollary 2. The algebras $\mathbb{T}^{\Gamma(p), p f}$ and $\mathbb{T}^{\Gamma, p f}$ are naturally isomorphic. The algebras $\mathbb{T}^{\Gamma(p)}$ and $\mathbb{T}^{\Gamma}$ are naturally isomorphic.

Proof. The natural restriction maps between the Hecke algebras of level $N p$ and $N$ are isomorphisms because their action is continuous and the modules on which they are acting have the same closure. See the proof of Cor. 13 of [8] for more details.

3.4. Full Hecke algebras. In this subsection, we consider full Hecke algebras i.e. the Hecke algebras generated by all the operators $T_{q}, S_{q}$ for primes $q$ with $(q, N p)=1, U_{\ell}$ for primes $\ell$ dividing $N$ and $U_{p}$. So, $\mathbb{T}_{k}^{\Gamma(p) \text {,full }}, D A_{k}^{\Gamma \text {,full }}$ and $A_{k}^{\Gamma \text {,full }}$ are the Hecke algebras generated by the Hecke operators $T_{q}, S_{q}$ for primes $q$ with $(q, N p)=1, U_{\ell}$ for primes $\ell$ dividing $N$ and $U_{p}$ acting on $D_{\leq k}^{\Gamma(p)}(\mathcal{O}), D_{\leq k}^{\Gamma}(\mathbb{F})$ and $S_{\leq k}^{\Gamma}(\mathbb{F})$, respectively. We denote by $\mathbb{T}^{\Gamma(p) \text {,full }}, D A^{\Gamma \text {,full }}$ and $A^{\Gamma \text {,full }}$ the full Hecke algebras acting on $D^{\Gamma(p)}(\mathcal{O}), D^{\Gamma}(\mathbb{F})$ and $S^{\Gamma}(\mathbb{F})$, respectively which are obtained by taking the inverse limits over the weights $k$ of appropriate Hecke algebras as before.

Proposition 3. The pairings $\mathbb{T}_{k}^{\Gamma(p), f u l l} \times D_{\leq k}^{\Gamma(p)}(\mathcal{O}) \rightarrow \mathcal{O}, D A_{k}^{\Gamma, f u l l} \times D_{\leq k}^{\Gamma}(\mathbb{F}) \rightarrow \mathbb{F}, A_{k}^{\Gamma, \text { full }} \times$ $S_{\leq k}^{\Gamma}(\mathbb{F}) \rightarrow \mathbb{F}$ given by $(t, f) \mapsto a_{1}(t f)$ are perfect.

Proof. This is well known but we recall the proof here. Suppose $f \in D_{\leq k}^{\Gamma(p)}(\mathcal{O})$ be such

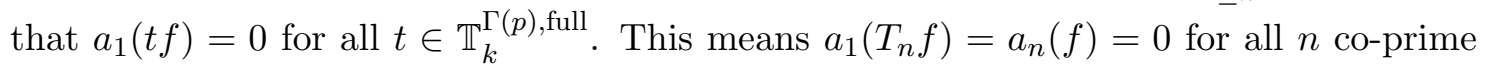
to $N p, a_{1}\left(U_{\ell} f\right)=a_{\ell}(f)=0$ for all primes $\ell$ dividing $N$ and $a_{1}\left(U_{p} f\right)=a_{p}(f)=0$. Thus, we have $a_{n}(f)=0$ for all $n$ which implies $f=0$. Now, suppose $t \in \mathbb{T}_{k}^{\Gamma(p) \text {,full }}$ is such that $a_{1}(t f)=0$ for all $f \in D_{\leq k}^{\Gamma(p)}(\mathcal{O})$. This means $a_{1}(s(t f))=a_{1}(t(s f))=0$ for all $s \in \mathbb{T}_{k}^{\Gamma(p) \text {,full }}$. Thus, from the previous part, we get that $t f=0$ for all $f \in D_{\leq k}^{\Gamma(p)}(\mathcal{O})$ which means $t=0$. The proof for other cases goes in the exact same way.

Corollary 3. The map $\mathbb{T}_{k}^{\Gamma(p), \text { full }} \rightarrow D A_{k}^{\Gamma, \text { full }}$ induces an isomorphism $\mathbb{T}_{k}^{\Gamma(p), f u l l} \otimes_{\mathcal{O}} \mathbb{F} \rightarrow$ $D A_{k}^{\Gamma, \text { full }}$. Hence, we get an isomorphism $\mathbb{T}^{\Gamma(p), f u l l} \otimes_{\mathcal{O}} \mathbb{F} \simeq D A^{\Gamma, \text { full }}$.

Proof. By the perfect duality above and Lemma 3, we see that the rank of torsion-free $\mathcal{O}$-module $\mathbb{T}_{k}^{\Gamma(p) \text {,full }}$ and the dimension of $D A_{k}^{\Gamma \text {,full }}$ as $\mathbb{F}$-vector space are the same which implies the corollary above. See the proof of Cor. 15 of [8] for more details.

The composition $\Lambda \rightarrow \mathbb{T}^{\Gamma, \mathrm{pf}} \rightarrow \mathbb{T}^{\Gamma(p) \text {,full }}$ defines a structure of $\Lambda$-algebra on $\mathbb{T}^{\Gamma(p), \text { full }}$.

Proposition 4. One has $\mathbb{T}^{\Gamma(p), f u l l} / m_{\Lambda} \mathbb{T}^{\Gamma(p), f u l l} \simeq A^{\Gamma, f u l l}$. 
Proof. The proposition follows from Theorem 4 (a theorem of Katz that we recalled in section 3.1), the perfect duality and its corollary above. See the proof of Prop. 16 of [8] for more details.

\section{Relation Between the components of Partial and Full Hecke algebras}

Throughout this section $\Gamma$ means either $\Gamma_{1}(N)$ or $\Gamma_{0}(N)$ and $\Gamma(p)$ means either $\Gamma_{1}(N p)$ or $\Gamma_{0}(N p)$ accordingly. Recall that we have a direct product decomposition $\mathbb{T}^{\Gamma}=\prod \mathbb{T}_{\bar{\rho}}^{\Gamma}$ and a direct sum decomposition $S^{\Gamma}(\mathcal{O})=\oplus S^{\Gamma}(\mathcal{O})_{\bar{\rho}}$ where the product and sum are taken over all the $\Gamma$-modular representations. Note that, $S^{\Gamma}(\mathcal{O})_{\bar{\rho}}$ is the intersection of the subspace of $S^{\Gamma}\left(\overline{\mathbb{Q}_{p}}\right)$ generated by the eigenforms lifting the system of eigenvalues corresponding to $\bar{\rho}$ with $S^{\Gamma}(\mathcal{O})$. The decompositions above are such that $\mathbb{T}_{\bar{\rho}}^{\Gamma}$ is also the largest quotient of $\mathbb{T}^{\Gamma}$ which acts faithfully on $S^{\Gamma}(\mathcal{O})_{\bar{\rho}}$. Let $\mathbb{T}_{\bar{\rho}}^{\Gamma \text {,pf }}$ be the largest quotient of $\mathbb{T}^{\Gamma, \mathrm{pf}}$ which acts faithfully on $S^{\Gamma}(\mathcal{O})_{\bar{\rho}}$. Define $A_{\bar{\rho}}^{\Gamma, \text { pf }}$ in a similar way.

Note that, since $D^{\Gamma}(\mathcal{O})$ contains $S^{\Gamma}(\mathcal{O})$ as a co-torsion submodule, we have a direct sum decomposition $D^{\Gamma}(\mathcal{O})=\oplus D^{\Gamma}(\mathcal{O})_{\bar{\rho}}$ similar to that of $S^{\Gamma}(\mathcal{O})$. Moreover, $\mathbb{T}_{\bar{\rho}}^{\Gamma, p f}$ and $\mathbb{T}_{\bar{\rho}}^{\Gamma}$ are the largest of quotients of $\mathbb{T}^{\Gamma, p f}$ and $\mathbb{T}^{\Gamma}$ respectively, acting faithfully on $D^{\Gamma}(\mathcal{O})_{\bar{\rho}}$.

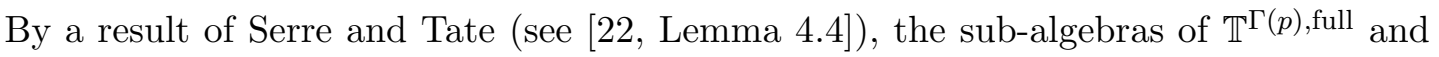
$A^{\Gamma \text {,full }}$ generated by the Hecke operators $T_{q}, S_{q}$ for primes $q$ not dividing $N p$ and $U_{p}$ are semi-local and the local components of both of them are in bijection with the set of $\mathbb{F}$-valued systems of eigenvalues of the Hecke operators $T_{q}, S_{q}$ for primes $q$ not dividing $N p$ and $U_{p}$ appearing in $S^{\Gamma}(\mathbb{F})$. Hence, they are in bijection with the pairs $(\bar{\rho}, \lambda)$, where $\bar{\rho}$ $: G_{\mathbb{Q}, N p} \rightarrow G L_{2}(\mathbb{F})$ is a $\Gamma$-modular representation attached to some eigenform $f \in S^{\Gamma}(\mathbb{F})$ and $\lambda$ is the eigenvalue of $U_{p}$ on $f$ (see [22]).

So, we get a direct sum decomposition $D^{\Gamma(p)}(\mathcal{O})=\oplus D^{\Gamma(p)}(\mathcal{O})_{\bar{\rho}, \lambda}$ similar to that of $S^{\Gamma}(\mathcal{O})$ seen above. Now, let us define $\mathbb{T}_{\bar{\rho}, \lambda}^{\Gamma(p) \text {,full }}$ to be the largest quotient of $\mathbb{T}^{\Gamma(p) \text {,full }}$ acting faithfully on $D^{\Gamma(p)}(\mathcal{O})_{\bar{\rho}, \lambda}$ and $A_{\bar{\rho}, \lambda}^{\Gamma, \text { full }}$ to be the largest quotient of $A^{\Gamma \text {,full }}$ acting faithfully on $S^{\Gamma}(\mathbb{F})_{\bar{\rho}, \lambda}$.

We get, using the Chinese Remainder Theorem and the definitions above, the product decompositions $\mathbb{T}^{\Gamma(p) \text {,full }}=\prod \mathbb{T}_{\bar{\rho}, \lambda}^{\Gamma(p), \text { full }}, \mathbb{T}^{\Gamma, \mathrm{pf}}=\prod \mathbb{T}_{\bar{\rho}}^{\Gamma, \mathrm{pf}}$. Similarly, we get product decompositions of $A^{\Gamma, \text { full }}$ and $A^{\Gamma, \mathrm{pf}}$. Here, the products are finite products as the pairs $(\bar{\rho}, \lambda)$ are finitely many.

Proposition 5. (1) For a $\Gamma$-modular representation $\bar{\rho}$, one has a natural isomorphism of $A_{\bar{\rho}}^{\Gamma, p f}$-algebras $A_{\bar{\rho}}^{\Gamma, p f}\left[\left[U_{p}\right]\right] \simeq A_{\bar{\rho}, 0}^{\Gamma, f u l l}$.

(2) For a $\Gamma$-modular representation $\bar{\rho}$, one has a natural isomorphism of $\mathbb{T}_{\bar{\rho}}^{\Gamma, p f}$-algebras $\mathbb{T}_{\bar{\rho}}^{\Gamma, p f}\left[\left[U_{p}\right]\right] \simeq \mathbb{T}_{\bar{\rho}, 0}^{\Gamma(p), f u l l}$. 
Proof. The first part of this proposition is proved by Jochnowitz (see the proof of Theorem 6.3 of [22]). The proof of the second part of the proposition is the same as that of Proposition 17 of [8]. In the proof of Proposition 17 in [8], they adapt the proof of Jochnowitz in characteristic 0 which relies on the interplay between the operators $U_{p}$ and $V$. Here, $V$ is the operator which sends $\sum a_{n} q^{n}$ to $\sum a_{n} q^{p n}$. The same argument works here.

\section{Finiteness of $\mathbb{T}_{\bar{\rho}}^{\Gamma, p f}$ OVER $\mathbb{T}_{\bar{\rho}}^{\Gamma}$ FOR NEW $\bar{\rho}$}

Throughout this section, $\Gamma$ means either $\Gamma_{1}(N)$ or $\Gamma_{0}(N)$ and $\Gamma(M)$ means either $\Gamma_{1}(M)$ or $\Gamma_{0}(M)$ accordingly. Let us call a $\Gamma$-modular representation $\bar{\rho}$ new if it is not $\Gamma(M)$-modular for any proper divisor $M$ of $N$. Thus, the system of eigenvalues of the Hecke operators corresponding to $\bar{\rho}$ does not have a nontrivial eigenspace in $S^{\Gamma(M)}(\mathbb{F})$ for

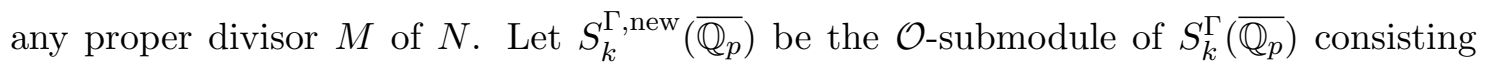
of new modular forms of weight $k$ and level $\Gamma$. Let $S_{\leq k}^{\Gamma, \text { new }}\left(\overline{\mathbb{Q}_{p}}\right)=\sum_{i=0}^{k} S_{i}^{\Gamma, \text { new }}\left(\overline{\mathbb{Q}_{p}}\right)$ and $S^{\Gamma, \text { new }}\left(\overline{\mathbb{Q}_{p}}\right)=\bigcup_{k=0}^{\infty} S_{\leq k}^{\Gamma, \text { new }}\left(\overline{\mathbb{Q}_{p}}\right)$. The following lemma follows directly from the discussion above and the description of $S^{\Gamma}(\mathcal{O})_{\bar{\rho}}$ given in the previous section:

Lemma 6. If $\bar{\rho}$ is a new $\Gamma$-modular representation, then $S^{\Gamma}(\mathcal{O})_{\bar{\rho}}$ is an $\mathcal{O}$-submodule of $S^{\Gamma, \text { new }}\left(\overline{\mathbb{Q}_{p}}\right)$.

Now we recall a well-known result regarding the Galois representations attached to level $N$ newforms:

Lemma 7. Let $f$ be a classical newform of tame level $N$ over $\overline{\mathbb{Q}_{p}}$. Let $\rho_{f}$ be the $p$-adic Galois representation attached to $f$ and let $V_{f}$ denote its underlying space. Let $\ell$ be a prime dividing $N$ and let $a_{\ell}(f)$ be the $U_{\ell}$ eigenvalue of $f$. Let $\left(V_{f}\right)_{I_{\ell}}$ be the vector space of $I_{\ell}$ co-invariants of $V_{f}$. Then the following are equivalent:

(1) $a_{\ell}(f)$ is a non-unit

(2) $a_{\ell}(f)=0$

(3) $\left(V_{f}\right)_{I_{\ell}}=0$

If these equivalent conditions do not hold, then we have that $\left(V_{f}\right)_{I_{\ell}}$ is one dimensional and $a_{\ell}(f)$ is equal to the eigenvalue of Frob $b_{\ell}$ acting on this line.

(See Lemma 2.6.1 of [18]).

Proposition 6. If $\bar{\rho}$ is a new $\Gamma$-modular representation, then $\mathbb{T}_{\bar{\rho}}^{\Gamma, p f}$ is finite over $\mathbb{T}_{\bar{\rho}}^{\Gamma}$. More precisely, $U_{\ell}$ (for every $\ell \mid N$ ) is integral over $\mathbb{T}_{\bar{\rho}}^{\Gamma}$ of degree at most 2 . If one of the following condition holds: 
(1) $\Gamma=\Gamma_{0}(N)$ and there does not exist a prime $\ell$ such that $\ell \| N$ and $p \mid \ell+1$,

(2) $\Gamma=\Gamma_{1}(N)$ and if there exists a prime $\ell$ such that $p \mid \ell^{2}-1$ and $\ell|| N$, then $p \mid \ell+1$ and $\operatorname{det} \bar{\rho}\left(I_{\ell}\right) \neq 1$

then $\mathbb{T}_{\bar{\rho}}^{\Gamma, p f}=\mathbb{T}_{\bar{\rho}}^{\Gamma}$

Proof. Let $\Gamma=\Gamma_{0}(N)$. Since $\bar{\rho}$ is new, we know, from Lemma 6 , that $S^{\Gamma}(\mathcal{O})_{\bar{\rho}} \subset$ $S^{\Gamma, \text { new }}\left(\overline{\mathbb{Q}_{p}}\right)$. Now, $\mathbb{T}_{\bar{\rho}}^{\Gamma, \text { pf }}$ is the largest quotient of $\mathbb{T}^{\Gamma, \text { pf }}$ which acts faithfully on $S^{\Gamma}(\mathcal{O})_{\bar{\rho}}$. Let $\ell$ be a prime dividing $N$. By Theorem 5 of [2], if $\ell^{2}$ divides $N$, then $U_{\ell}$ acts like 0 on $S_{k}^{\Gamma, \text { new }}\left(\overline{\mathbb{Q}_{p}}\right)$ and if $\ell \| N$, then $U_{\ell}^{2}$ acts like $\ell^{k-2}$ on $S_{k}^{\Gamma, n e w}\left(\overline{\mathbb{Q}_{p}}\right)$. Hence, if $\ell^{2}$ divides $N$, then $U_{\ell}$ acts like 0 on $S^{\Gamma}(\mathcal{O})_{\bar{\rho}}$ and if $\ell \| N$, then $U_{\ell}^{2}$ acts like $\ell^{-2} \phi(\ell)$ on $S^{\Gamma}(\mathcal{O})_{\bar{\rho}}$, where $\phi$ $: \mathbb{Z}_{p}^{*} \rightarrow \mathbb{T}_{\bar{\rho}}^{\Gamma}$ is the map considered in Lemma 5. Thus, if $\ell^{2} \mid N$, then $U_{\ell}=0$, and if $\ell \| N$, then $U_{\ell}^{2}-\ell^{-2} \phi(\ell)=0$ in $\mathbb{T}_{\bar{\rho}}^{\Gamma, p f}$.

Suppose $\ell \| N$ and let $f$ be a newform of level $\Gamma_{0}(N)$ lifting the system of eigenvalues corresponding to $\bar{\rho}$. Let $\pi_{\ell}$ be the $\ell$-component of the automorphic representation corresponding to $f$ and $\rho_{f}$ be the $p$-adic Galois representation attached to $f$. As $\ell^{2} \nmid N$, $\pi_{\ell}$ is either principal series or special (see section 1.2 of [11]). So, it follows from the local Langlands correspondence, that $\left.\rho_{f}\right|_{G_{\mathbb{Q}_{\ell}}}$ is either a direct sum of two characters or a non-trivial extension of a character by its cyclotomic twist (see section 3 and 5 of [31]). As $f$ is a newform of level $\Gamma$, the Artin conductor of $\rho_{f}$ is $N$ (level of $f$ ). Since $\ell \| N$, the exponent of $\ell$ appearing in the Artin conductor of $\rho_{f}$ is exactly 1 which means $\left(\rho_{f}\right)^{I_{\ell}}$, the subspace of $\rho_{f}$ on which $I_{\ell}$ acts trivially, is one dimensional. So, if $\left.\rho_{f}\right|_{G_{\mathbb{Q}_{\ell}}}$ is a direct sum of two characters, then one of them is unramified and the other is tamely ramified. Otherwise, $\left.\rho_{f}\right|_{G_{\mathbb{Q}_{\ell}}}$ is a non-trivial extension of an unramified character by its cyclotomic twist. As $f$ is a modular form of level $\Gamma_{0}(N)$, its nebentypus is trivial, which means $\operatorname{det} \rho_{f}\left(I_{\ell}\right)=1$. This implies that $\left.\rho_{f}\right|_{G_{\mathbb{Q}_{\ell}}}$ is not a direct sum of two characters and hence, $\left.\rho_{f}\right|_{G_{\mathbb{Q}_{\ell}}} \simeq\left(\begin{array}{cc}\epsilon_{p} \chi & * \\ 0 & \chi\end{array}\right)$, where $*$ is non-zero and ramified, $\chi$ is an unramified character and $\epsilon_{p}$ is the $p$-adic cyclotomic character of $G_{\mathbb{Q}_{\ell}}$.

As $a_{\ell}(f)$, the $U_{\ell}$ eigenvalue of $f$, is non-zero, by Lemma 7 above, it is the eigenvalue of Frob ${ }_{\ell}$ acting on $\left(\rho_{f}\right)_{I_{\ell}}$. Thus, $a_{\ell}(f)=\chi\left(\right.$ Frob $\left._{\ell}\right)$. Let $x$ be a lift of Frob ${ }_{\ell}$ in $G_{\mathbb{Q}_{\ell}}$. Note that, $\operatorname{tr}\left(\rho_{f} \circ i_{\ell}(x)\right)=\epsilon_{p} \chi\left(\right.$ Frob $\left._{\ell}\right)+\chi\left(\right.$ Frob $\left._{\ell}\right)=(\ell+1) \chi\left(\operatorname{Frob}_{\ell}\right)=(\ell+1) a_{\ell}(f)$, which means $a_{\ell}(f)=\frac{\operatorname{tr}\left(\rho_{f} \circ i_{\ell}(x)\right)}{\ell+1}$. Suppose $p \nmid \ell+1$ which implies that $\ell+1$ is a unit in $\mathbb{T}_{\bar{\rho}}^{\Gamma}$. Then on every newform $f$ of level $\Gamma$ lifting $\bar{\rho}$, the action of $U_{\ell}$ coincides with the action of $\frac{\left(\tau^{\Gamma} \circ i_{\ell}\right)(x)}{\ell+1}$ which lies in $\mathbb{T}_{\bar{\rho}}^{\Gamma}$ as $\ell+1$ is a unit in $\mathbb{T}_{\bar{\rho}}^{\Gamma}$. As $\bar{\rho}$ is new, every eigenform of level $\Gamma$ lifting $\bar{\rho}$ is a newform. This implies that $U_{\ell}-\frac{\left(\tau^{\Gamma} \circ i_{\ell}\right)(x)}{\ell+1}$ acts like 0 on $S^{\Gamma}(\mathcal{O})_{\bar{\rho}}$ and hence, $U_{\ell}=\frac{\left(\tau^{\Gamma} \circ i_{\ell}\right)(x)}{\ell+1}$ in $\mathbb{T}_{\bar{\rho}}^{\Gamma, p f}$. Therefore, $U_{\ell} \in \mathbb{T}_{\bar{\rho}}^{\Gamma}$ if $\ell \| N$ and $p \nmid \ell+1$. 
Note that, $\mathbb{T}_{\bar{\rho}}^{\Gamma, p f}$ is generated by the Hecke operators $U_{\ell}$ over $\mathbb{T}_{\bar{\rho}}^{\Gamma}$. So, by combining the discussion of the last two paragraphs, we get that $\mathbb{T}_{\bar{\rho}}^{\Gamma, \mathrm{pf}}=\mathbb{T}_{\bar{\rho}}^{\Gamma}$ if there does not exist a prime $\ell$ such that $\ell \| N$ and $p \mid \ell+1$. Otherwise, we have $U_{\ell} \in \mathbb{T}_{\bar{\rho}}^{\Gamma}$ if either $\ell^{2} \mid N$ or $p \nmid \ell+1$ and $U_{\ell}^{2}-\ell^{-2} \phi(\ell)=0$ if $\ell \| N$. Therefore, we conclude that $\mathbb{T}_{\bar{\rho}}^{\Gamma, p f}$ is a finite extension of $\mathbb{T}_{\bar{\rho}}^{\Gamma}$.

Let $\Gamma=\Gamma_{1}(N)$. There is a continuous pseudo-representation $\left(\tau^{\Gamma}, \delta^{\Gamma}\right): G_{\mathbb{Q}, N p} \rightarrow \mathbb{T}_{\bar{\rho}}^{\Gamma}$ such that $\tau^{\Gamma}\left(\operatorname{Frob}_{q}\right)=T_{q}, \delta^{\Gamma}\left(\operatorname{Frob}_{q}\right)=q S_{q}$ for primes $q$ not dividing $N p$. Hence, we get a continuous pseudo-representation $(t, d): G_{\mathbb{Q}, N p} \rightarrow \mathbb{T}_{\bar{\rho}}^{\Gamma, p f}$ since $\mathbb{T}_{\bar{\rho}}^{\Gamma} \subset \mathbb{T}_{\bar{\rho}}^{\Gamma, p f}$. For every prime $\ell$ dividing $N$, let us choose an element $g_{\ell}$ of $G_{\mathbb{Q}_{\ell}}$ which gets mapped to Frob under the quotient map $G_{\mathbb{Q}_{\ell}} \rightarrow G_{\mathbb{Q}_{\ell}} / I_{\ell}$. We have already fixed a natural map $i_{\ell}: G_{\mathbb{Q}_{\ell}} \rightarrow G_{\mathbb{Q}, N p}$. Hence, we get a pseudo-representation $\left(t \circ i_{\ell}, d \circ i_{\ell}\right): G_{\mathbb{Q}_{\ell}} \rightarrow \mathbb{T}_{\bar{\rho}}^{\Gamma_{1}(N), \mathrm{pf}}$.

Now, consider the characteristic polynomial $Q_{\ell}(x)$ of $g_{\ell}$ which is defined by $Q_{\ell}(x)=$ $x^{2}-\left(t \circ i_{\ell}\right)\left(g_{\ell}\right) x+\left(d \circ i_{\ell}\right)\left(g_{\ell}\right)$. Let $f$ be a newform of level $N$ lifting the system of eigenvalues corresponding to $\bar{\rho}$. Denote by $\rho_{f}$ the $p$-adic Galois representation attached to $f$ and by $a_{\ell}(f)$ its $U_{\ell}$ eigenvalue. By Lemma 7 , if $a_{\ell}(f)=0$, then $U_{\ell}$ kills $f$. If $a_{\ell}(f) \neq 0$, then $a_{\ell}(f)$ is the root of the characteristic polynomial $P_{f}(x)$ of $\rho_{f} \circ i_{\ell}\left(g_{\ell}\right)$. But $Q_{\ell}\left(U_{\ell}\right) f=P_{f}\left(a_{\ell}(f)\right) f=0$. Thus, in this case, $Q_{\ell}\left(U_{\ell}\right)$ kills $f$. We will now determine if it is possible to have two newforms $f$ and $g$ of level $N$ lifting $\bar{\rho}$ such that $a_{\ell}(f) \neq 0$ but $a_{\ell}(g)=0$ or equivalently (by Lemma 7$),\left(\rho_{f}\right)_{I_{\ell}}$ is one dimensional but $\left(\rho_{g}\right)_{I_{\ell}}=0$.

Let $f$ be a newform of level $\Gamma$ lifting $\bar{\rho}$ as above and $\pi_{\ell}$ be the $\ell$-component of the automorphic representation corresponding to $f$. So, $\pi_{\ell}$ is one of the following: principal series, special and supercuspidal (see section 3 of [31]). As $f$ is a newform, the Artin conductor of $\rho_{f}$ is $N$ (level of $f$ ). Suppose $\ell \mid N$ but $\ell^{2} \nmid N$. Then, from the analysis carried out in the $\Gamma_{0}(N)$ case above, we see that $\left.\rho_{f}\right|_{G_{\mathbb{Q}_{\ell}}}$ is either a sum of an unramified character and a tamely ramified character or a non-trivial extension of an unramified character by its cyclotomic twist. Hence, the space $\left(\rho_{f}\right)_{I_{\ell}}$ of $I_{\ell}$ co-invariants is also one dimensional. Therefore, if $\ell \| N$, then for every newform $f$ of level $\Gamma$ lifting $\bar{\rho},\left(\rho_{f}\right)_{I_{\ell}}$ is one dimensional and hence, $a_{\ell}(f) \neq 0$.

Now suppose $\ell^{2} \mid N$ and moreover, the space of $I_{\ell}$ co-invariants of $\rho_{f}$ is one dimensional. So, the exponent of $\ell$ appearing in the Artin conductor of $\rho_{f}$ is at least 2. This means that $\pi_{\ell}$ is not special as otherwise, $\left(\rho_{f}\right)_{I_{\ell}}$ being one dimensional will imply that $\left.\rho_{f}\right|_{G_{\mathbb{Q}_{\ell}}}$ is a non-trivial extension of an unramified character by its cyclotomic twist and hence, the exponent of $\ell$ appearing in the Artin conductor of $\rho_{f}$ is 1 . The non-triviality of $\left(\rho_{f}\right)_{I_{\ell}}$ also implies that $\pi_{\ell}$ is not extraordinary supercuspidal (see proof of [31, Proposition 3.2] for more details). Suppose $\pi_{\ell}$ is supercuspidal but not extraordinary. Then, by the local Langlands correspondence, $\left.\rho_{f}\right|_{G_{\mathbb{Q}_{\ell}}}=\operatorname{Ind}_{G_{K}}^{G_{\mathbb{Q}_{\ell}}} \chi$, where $K$ is a quadratic extension of $\mathbb{Q}_{\ell}$, 
$G_{K}$ is the absolute Galois group of $K, \chi$ is a character of $G_{K}$ taking values in $\overline{\mathbb{Q}_{p}}$ and moreover, $\rho_{f}$ is irreducible (see section 3 of [31]). But as $\left(\rho_{f}\right)_{I_{\ell}}$ is one dimensional, we get that $\chi$ is an unramified character of $G_{K}$. However, since the maximal unramified extension of $K$ is an abelian extension of $\mathbb{Q}_{\ell}$, this implies that $\left.\rho_{f}\right|_{G_{\mathbb{Q}_{\ell}}}$ is a sum of two characters contradicting the hypothesis that $\left.\rho_{f}\right|_{G_{\mathbb{Q}_{\ell}}}$ is irreducible. Hence, $\pi_{\ell}$ is not supercuspidal. Therefore, $\pi_{\ell}$ is principal series which means that $\left.\rho_{f}\right|_{G_{\mathbb{Q}_{\ell}}}$ is a sum of two characters $\chi_{1}$ and $\chi_{2}$. Moreover, $\left(\rho_{f}\right)_{I_{\ell}} \neq 0$ implies that one of them is unramified, while $\ell^{2} \mid N$ implies that the other is wildly ramified. Without loss of generality, suppose $\chi_{1}$ is wildly ramified and $\chi_{2}$ is unramified.

Thus, $\left.\bar{\rho}\right|_{G_{\mathbb{Q}_{\ell}}}=\overline{\chi_{1}} \oplus \overline{\chi_{2}}$, where $\overline{\chi_{1}}$ and $\overline{\chi_{2}}$ are the reductions of $\chi_{1}$ and $\chi_{2}$ in characteristic $p$, respectively. Note that, $\overline{\chi_{2}}$ is unramified while $\overline{\chi_{1}}$ is wildly ramified as $\ell \neq p$. Let $g$ be another newform of level $\Gamma$ lifting $\bar{\rho}$ and $\pi_{\ell}^{\prime}$ be the $\ell$-component of the automorphic representation corresponding to $g$. If $\pi_{\ell}^{\prime}$ is special, then $\left.\rho_{g}\right|_{G_{\mathbb{Q}_{\ell}}}$ is a non-trivial extension of a character by the cyclotomic twist of itself. This would imply that both $\overline{\chi_{1}}$ and $\overline{\chi_{2}}$ are either unramified or ramified which is not the case. Hence, $\pi_{\ell}^{\prime}$ is not special. If $\pi_{\ell}^{\prime}$ is extraordinary supercuspidal, then $\left.\bar{\rho}\right|_{I_{\ell}}$ is irreducible as $p \geq 5$ (see proof of [31, Proposition 3.2]). So, $\pi_{\ell}^{\prime}$ is not extraordinary supercuspidal. If $\pi_{\ell}^{\prime}$ is supercuspidal but not extraordinary, then $\left.\rho_{g}\right|_{G_{\mathbb{Q}_{\ell}}}$ is induced from a character of the absolute Galois group of a quadratic extension of $\mathbb{Q}_{\ell}$. Moreover, the subspace of $\rho_{g}$ fixed by $I_{\ell}$ is trivial. But the subspace of $\bar{\rho}$ fixed by $I_{\ell}$ is one dimensional. Thus, the exponent of $\ell$ in the Artin conductor of $\rho_{g}$ is greater than the exponent of $\ell$ in the Artin conductor of $\bar{\rho}$ (see section 1.1 of [11]). But [11, Proposition 2], along with the assumption that $\pi_{\ell}^{\prime}$ is supercuspidal, implies that $\bar{\rho}$ is unramified at $\ell$ which gives us a contradiction. Therefore, $\pi_{\ell}^{\prime}$ is not supercuspidal.

This means that $\pi_{\ell}^{\prime}$ is principal series and hence, $\left.\rho_{g}\right|_{G_{\mathbb{Q}_{\ell}}}$ is a sum of two characters, say $\chi_{1}^{\prime}$ and $\chi_{2}^{\prime}$. Without loss of generality, suppose $\chi_{1}^{\prime}$ is a lift of $\overline{\chi_{1}}$ and $\chi_{2}^{\prime}$ is a lift of $\overline{\chi_{2}}$. As $\overline{\chi_{1}}$ is wildly ramified, the Artin conductor of $\overline{\chi_{1}}$ is same as the Artin conductor of $\chi_{1}^{\prime}$ and the Artin conductor of $\chi_{1}$ (see section 1.2 of [11]). As the exponent of $\ell$ in the Artin conductor of $\rho_{f}$ is the sum of the exponents of $\ell$ in the Artin conductors of $\chi_{1}$ and $\chi_{2}$ and its exponent in the Artin conductor of $\rho_{g}$ is the sum of its exponents in the Artin conductors of $\chi_{1}^{\prime}$ and $\chi_{2}^{\prime}$. As both $f$ and $g$ are newforms of level $N$, the exponents of $\ell$ in the Artin conductors of $\rho_{f}$ and $\rho_{g}$ are same. This implies that the Artin conductors of $\chi_{2}$ and $\chi_{2}^{\prime}$ are same, which means that $\chi_{2}^{\prime}$ is also unramified. In particular, we see that $\left(\rho_{g}\right)_{I_{\ell}}$ is one dimensional. As a consequence, we see that if $f$ is a newform of level $\Gamma$ lifting $\bar{\rho}$ and $\left(\rho_{f}\right)_{I_{\ell}}$ is one dimensional, then, for every newform $g$ of level $\Gamma$ lifting $\bar{\rho},\left(\rho_{g}\right)_{I_{\ell}}$ is one dimensional. 
Let us continue with the assumption that there is a newform $f_{0}$ of level $\Gamma$ lifting $\bar{\rho}$ such that $\left(\rho_{f_{0}}\right)_{I_{\ell}}$ is one dimensional. Observe that in this case, from the discussion so far, we get that for every newform $g$ of level $\Gamma$ lifting $\bar{\rho},\left.\rho_{g}\right|_{G_{\mathbb{Q}_{\ell}}}$ is reducible and moreover, at least one character appearing in the semi-simplification of $\left.\rho_{g}\right|_{G_{\mathbb{Q}_{\ell}}}$ is unramified. Thus, $\left(\left.\bar{\rho}\right|_{G_{\mathbb{Q}_{\ell}}}\right)^{\mathrm{ss}}=\alpha \oplus \beta$, where $\left(\left.\bar{\rho}\right|_{G_{\mathbb{Q}_{\ell}}}\right)^{\mathrm{ss}}$ is the semi-simplification of $\left.\bar{\rho}\right|_{G_{\mathbb{Q}_{\ell}}}$ and $\alpha$ and $\beta$ are characters of $G_{\mathbb{Q}_{\ell}}$ such that at least one of them is unramified. Note that, both $\alpha$ and $\beta$ are defined over $\mathbb{F}$. Indeed, both $\left.\alpha\right|_{I_{\ell}}$ and $\left.\beta\right|_{I_{\ell}}$ take values in $\mathbb{F}$ as one of them is unramified and $\operatorname{det}(\bar{\rho})$ is defined over $\mathbb{F}$. So it follows from the previous discussion that the image of $I_{\ell}$ under $\bar{\rho}$ in $G L_{2}(\mathbb{F})$ is abelian and hence, is upper-triangular under a suitable basis. Therefore, since $I_{\ell}$ is normal in $G_{\mathbb{Q}_{\ell}}$, it follows that if $\bar{\rho}$ is ramified at $\ell$, then under the same basis, the image of $G_{\mathbb{Q}_{\ell}}$ under $\bar{\rho}$ in $G L_{2}(\mathbb{F})$ is also upper-triangular which implies that both $\alpha$ and $\beta$ are defined over $\mathbb{F}$. If $\bar{\rho}$ is unramified at $\ell$, then $\bar{\rho}$ is reducible as it is new. As $\bar{\rho}$ is semi-simple, it follows that both $\alpha$ and $\beta$ are defined over $\mathbb{F}$.

Suppose $\alpha \neq \beta$. Then, by [6, Theorem 1.4.4, Chapter 1], $\mathbb{T}_{\bar{\rho}}^{\Gamma}\left[G_{\mathbb{Q}_{\ell}}\right] /\left(\operatorname{ker}\left(\tau^{\Gamma} \circ i_{\ell}\left(G_{\mathbb{Q}_{\ell}}\right)\right)\right)$ is a Generalized Matrix Algebra (GMA) of the form $\left(\begin{array}{cc}\mathbb{T}_{\bar{\rho}}^{\Gamma} & B \\ C & \mathbb{T}_{\bar{\rho}}^{\Gamma}\end{array}\right)$, where $B$ and $C$ are finitely generated $\mathbb{T}_{\bar{\rho}}^{\Gamma}$-modules contained in the total fraction ring of $\mathbb{T}_{\bar{\rho}}^{\Gamma}$, and the diagonal entries reduce to $\alpha$ and $\beta$ modulo the maximal ideal of $\mathbb{T}_{\bar{\rho}}^{\Gamma}$. Moreover, $B C \subset \mathbb{T}_{\bar{\rho}}^{\Gamma}$ and it is an ideal of $\mathbb{T}_{\bar{\rho}}^{\Gamma}$. Let us call it $I$.

For a newform $f$ of level $\Gamma$ lifting $\bar{\rho}$, let $\phi_{f}: \mathbb{T}_{\bar{\rho}}^{\Gamma} \rightarrow \overline{\mathbb{Q}_{p}}$ be the map which sends a Hecke operator to its $f$-eigenvalue and denote its kernel by $P_{f}$. From the previous paragraph, we see that the 2 dimensional pseudocharacter $\tau^{\Gamma} \circ i_{\ell}$ of $G_{\mathbb{Q}_{\ell}}$ is a sum of two characters modulo $P_{f}$ and hence, is reducible modulo $P_{f}$ for every newform $f$ of level $\Gamma$ lifting $\bar{\rho}$. Therefore, by [6, Proposition 1.5.1, Chapter 1], it follows that $I \subset P_{f}$ for every newform $f$ of level $\Gamma$ lifting $\bar{\rho}$. This means that if $x \in I$, then $x(f)=0$ for every newform $f$ of level $\Gamma$ lifting $\bar{\rho}$. As every eigenform of level $\Gamma$ lifting $\bar{\rho}$ is a newform, we see that $I=0$. Thus, the projection on the diagonal entries of the GMA $\left(\begin{array}{cc}\mathbb{T}_{\bar{\rho}}^{\Gamma} & B \\ C & \mathbb{T} \\ \overline{\bar{\rho}}\end{array}\right)$ above gives two characters $\tilde{\alpha}$ and $\tilde{\beta}$ of $G_{\mathbb{Q}_{\ell}}$ taking values in $\mathbb{T}_{\bar{\rho}}^{\Gamma}$ such that, $\tilde{\alpha}$ is a deformation of $\alpha$, while $\tilde{\beta}$ is a deformation of $\beta$.

As at least one of $\alpha$ and $\beta$ is unramified, without loss of generality, assume that $\beta$ is unramified. Suppose $\alpha$ is ramified. Then, it follows, from the analysis above, that for a newform $f$ of level $\Gamma$ lifting $\bar{\rho},\left.\rho_{f}\right|_{G_{\mathbb{Q}_{\ell}}}$ is a direct sum of an unramified character and a ramified character. As $\alpha$ is ramified, the unramified character appearing in $\left.\rho_{f}\right|_{G_{\mathbb{Q}_{\ell}}}$ is a lift of $\beta$ and hence, it is the image of $\tilde{\beta}$ modulo $P_{f}$. This means that the reduction of $\tilde{\beta}$ modulo $P_{f}$ gives an unramified character for every newform $f$ of level $\Gamma$ lifting $\bar{\rho}$. Hence, by the reasoning used in the previous paragraph, we see that $\tilde{\beta}$ is an unramified character of $G_{\mathbb{Q}_{\ell}}$. By Lemma 7 , it follows that for every newform $f$ of level $\Gamma$ lifting $\bar{\rho}$, the $U_{\ell}$ 
eigenvalue of $f$ is the reduction of $\tilde{\beta}\left(\right.$ Frob $\left._{\ell}\right)$ modulo $P_{f}$. Thus, $U_{\ell}-\tilde{\beta}\left(\right.$ Frob $\left._{\ell}\right)$ annihilates every newform $f$ of level $\Gamma$ lifting $\bar{\rho}$. Therefore, $U_{\ell}=\tilde{\beta}\left(\right.$ Frob $\left._{\ell}\right)$ in $\mathbb{T}_{\bar{\rho}}^{\Gamma, p f}$ and hence, $U_{\ell} \in \mathbb{T}_{\bar{\rho}}^{\Gamma}$ as $\tilde{\beta}\left(\right.$ Frob $\left._{\ell}\right) \in \mathbb{T}_{\bar{\rho}}^{\Gamma}$.

Now, suppose $\alpha$ is also unramified. This means that for a newform $f$ of level $\Gamma$ lifting $\bar{\rho},\left.\rho_{f}\right|_{G_{\mathbb{Q}_{\ell}}}$ is either a non-trivial extension of an unramified character by its cyclotomic twist or a direct sum of an unramified character and a tamely ramified character and in both cases, $\ell \| N$ (see [11, Proposition 2]). Moreover, in the second case, $p \mid \ell-1$. Suppose $\left.\rho_{f}\right|_{G_{\mathbb{Q}_{\ell}}}$ is a direct sum of an unramified character and a tamely ramified character. Let $\epsilon$ be the nebentypus of $f$. Note that, $\operatorname{det}\left(\rho_{f}\left(I_{\ell}\right)\right)=\epsilon\left((\mathbb{Z} / \ell \mathbb{Z})^{*}\right) \neq 1$ but its reduction is 1 in characteristic $p$. So, by [11, Proposition 3], there exists a newform $g$ of level $\Gamma$ lifting $\bar{\rho}$ such that $\epsilon^{\prime}\left((\mathbb{Z} / \ell \mathbb{Z})^{*}\right)=1$, where $\epsilon^{\prime}$ is the nebentypus of $g$. Thus, $\left.\rho_{g}\right|_{G_{\mathbb{Q}_{\ell}}}$ is a non-trivial extension of an unramified character by its cyclotomic twist. This means that either $\alpha / \beta$ or $\beta / \alpha$ is the cyclotomic character $\omega_{p}$. But as $p \mid \ell-1, \omega_{p}\left(\right.$ Frob $\left._{\ell}\right)=1$ and hence, $\omega_{p}$ is the trivial character. However, this means that $\alpha=\beta$ which contradicts our assumption that $\alpha \neq \beta$. Therefore, we get that, if $\alpha$ is unramified, then $\left.\rho_{f}\right|_{G_{\mathbb{Q}_{\ell}}}$ is a non-trivial extension of an unramified character by the cyclotomic twist of itself for every newform $f$ of level $\Gamma$ lifting $\bar{\rho}$. By [11, Proposition 3] and the discussion above, it follows that $p \nmid \ell-1$. Indeed, if $p \mid \ell-1$, then by [11, Proposition 3], there exists a newform $g$ lifting $\bar{\rho}$ such that $\operatorname{det}\left(\rho_{g}\left(I_{\ell}\right)\right) \neq 1$ which gives a contradiction.

As $\alpha$ is also unramified, we saw above that either $\alpha / \beta$ or $\beta / \alpha$ is $\omega_{p}$. If both of them are $\omega_{p}$, then $\omega_{p}^{2}=1$ which means $p \mid \ell^{2}-1$. As $p \nmid \ell-1$, we get that $p \mid \ell+1$ if both of them are $\omega_{p}$. Suppose $p \nmid \ell+1$ which implies that exactly one of them is $\omega_{p}$. Without loss of generality, assume $\alpha / \beta$ is $\omega_{p}$. Now in this case, the image of $\tilde{\alpha}$ is the cyclotomic twist of the image of $\tilde{\beta}$ modulo $P_{f}$ for every newform $f$ of level $\Gamma$ lifting $\bar{\rho}$. Thus, for every such newform $f, G_{\mathbb{Q}_{\ell}}$ acts by the image of $\tilde{\beta}$ modulo $P_{f}$ on $\left(\rho_{f}\right)_{I_{\ell}}$. Hence, the $U_{\ell}$ eigenvalue of $f$ is the reduction of $\tilde{\beta}\left(\right.$ Frob $\left._{\ell}\right)$ modulo $P_{f}$ for every newform $f$ of level $\Gamma$ lifting $\bar{\rho}$. Therefore, by the reasoning used in the previous case, we see that $U_{\ell}=\tilde{\beta}\left(\right.$ Frob $\left._{\ell}\right)$ in $\mathbb{T}_{\bar{\rho}}^{\Gamma, p f}$ which means that $U_{\ell} \in \mathbb{T}_{\bar{\rho}}^{\Gamma}$.

Now suppose that there exists a newform $f_{0}$ of level $\Gamma$ lifting $\bar{\rho}$ such that $\left(\rho_{f_{0}}\right)_{I_{\ell}}=0$ which means $U_{\ell} f_{0}=0$. Then, by our analysis above, it follows that $\ell^{2} \mid N$ and $U_{\ell} f=0$ for all newforms $f$ of level $\Gamma$ lifting $\bar{\rho}$. As $\bar{\rho}$ is new of level $\Gamma$, we get, by the reasoning used above, that $U_{\ell}=0$ in $\mathbb{T}_{\bar{\rho}}^{\Gamma, p f}$.

By combining the discussion so far, we see that for a prime $\ell$ dividing $N, U_{\ell} \in \mathbb{T} \overline{\bar{\rho}}$ if one of the following conditions hold:

(1) $\ell^{2} \mid N$

(2) $\ell \| N$ and $p \nmid \ell^{2}-1$, 
(3) $\ell|| N, p \mid \ell+1$ and $\operatorname{det} \bar{\rho}\left(I_{\ell}\right) \neq 1$.

Otherwise, $Q_{\ell}\left(U_{\ell}\right)$ kills every newform $f$ of level $N$ lifting $\bar{\rho}$. Since $\bar{\rho}$ is new, every eigenform of level $N$ lifting $\bar{\rho}$ is a newform. This implies that either $U_{\ell} \in \mathbb{T}_{\bar{\rho}}^{\Gamma}$ or $Q_{\ell}\left(U_{\ell}\right)=0$ for every prime $\ell$ dividing $N$. For every prime $\ell$ dividing $N, Q_{\ell}(x)$ is a monic polynomial with coefficients in $\mathbb{T}_{\bar{\rho}}^{\Gamma}$ as the pseudo-representation $(t, d)$ takes values in $\mathbb{T}_{\bar{\rho}}^{\Gamma}$. Hence, $U_{\ell}$ is integral over $\mathbb{T}_{\bar{\rho}}^{\Gamma}$ for every prime $\ell$ dividing $N$ of degree at most 2 . Therefore, $\mathbb{T}_{\bar{\rho}}^{\Gamma, p f}$ is finite over $\mathbb{T}_{\bar{\rho}}^{\Gamma}$ and moreover, $\mathbb{T}_{\bar{\rho}}^{\Gamma, p f}=\mathbb{T}_{\bar{\rho}}^{\Gamma}$ if $\ell|| N$ and $p \mid \ell^{2}-1$ implies that $p \mid \ell+1$ and $\operatorname{det} \bar{\rho}\left(I_{\ell}\right) \neq 1$ as $\mathbb{T}_{\bar{\rho}}^{\Gamma, p f}$ is generated by these $U_{\ell}$ 's over $\mathbb{T}_{\bar{\rho}}^{\Gamma}$.

Remark. (1) Note that, the proof given above for the $\Gamma_{1}(N)$ case works for the $\Gamma_{0}(N)$ case as well. But we give a different proof because it gives us a more precise result in the $\Gamma_{0}(N)$ case and it is also simpler than the proof in the $\Gamma_{1}(N)$ case.

(2) Even though we do a detailed analysis in the $\Gamma_{1}(N)$ case above, it is not really necessary to only prove that $U_{\ell}$ integral over $\mathbb{T}_{\bar{\rho}}^{\Gamma_{1}(N)}$. Indeed, by the reasoning used in the last paragraph of the proof above, we can easily prove that $U_{\ell} Q_{\ell}\left(U_{\ell}\right)=0$ which proves that $U_{\ell}$ is integral over $\mathbb{T}_{\bar{\rho}}^{\Gamma_{1}(N)}$. For most of our purposes, we only need the result that $U_{\ell}$ is integral over $\mathbb{T}_{\bar{\rho}}^{\Gamma_{1}(N)}$. But we give this detailed analysis to obtain a more precise result which is helpful in getting more precise version of Theorem 3 in some cases (see the remark after the proof of Theorem 3 ).

\section{The CASE OF $\bar{\rho}$ Which IS NOT NEW}

Throughout this section $\Gamma$ means either $\Gamma_{1}(N)$ or $\Gamma_{0}(N)$ and $\Gamma(M)$ means either $\Gamma_{1}(M)$ or $\Gamma_{0}(M)$ accordingly. Let $\bar{\rho}$ be a $\Gamma$-modular representation which is not new. Thus, the system of $\mathbb{F}$-valued eigenvalues corresponding to $\bar{\rho}$ has a nontrivial eigenspace in $S^{\Gamma(M)}(\mathbb{F})$ for some proper divisor $M$ of $N$. Let us denote by $M_{\bar{\rho}}$ the smallest divisor of $N$ such that the system of $\mathbb{F}$-valued eigenvalues corresponding to $\bar{\rho}$ has a nontrivial eigenspace in $S^{\Gamma\left(M_{\bar{\rho}}\right)}(\mathbb{F})$. Note that, $\bar{\rho}$ is then a new $\Gamma\left(M_{\bar{\rho}}\right)$-modular representation.

Lemma 8. If $\bar{\rho}$ is a $\Gamma$-modular representation which is not new, then the natural map of local algebras $r: A_{\bar{\rho}}^{\Gamma} \rightarrow A_{\bar{\rho}}^{\Gamma\left(M_{\bar{\rho}}\right)}$ obtained by restriction is surjective.

Proof. First note that $S^{\Gamma\left(M_{\bar{\rho}}\right)}(\mathbb{F})_{\bar{\rho}} \subset S^{\Gamma}(\mathbb{F})_{\bar{\rho}}$. Hence, using this inclusion, we obtain a natural map $r: A_{\bar{\rho}}^{\Gamma} \rightarrow A_{\bar{\rho}}^{\Gamma\left(M_{\bar{\rho}}\right)}$ by restriction. Its image $\operatorname{Im}(r)$ is complete and hence, closed. It contains all the Hecke operators $T_{q}$ and $q S_{q}$ (considered as operators on $\left.S^{\Gamma\left(M_{\bar{\rho}}\right)}(\mathbb{F})_{\bar{\rho}}\right)$ for all the primes $q$ not dividing $N p$. There is a continuous pseudorepresentation $\left(\tilde{\tau}^{\Gamma\left(M_{\bar{\rho}}\right)}, \tilde{\delta}^{\Gamma\left(M_{\bar{\rho}}\right)}\right): G_{\mathbb{Q}, M_{\bar{\rho}} p} \rightarrow A_{\bar{\rho}}^{\Gamma\left(M_{\bar{\rho}}\right)}$ and $\operatorname{Im}(r)$ contains the Hecke operators $T_{q}=\tilde{\tau}^{\Gamma\left(M_{\bar{\rho}}\right)}\left(\operatorname{Frob}_{q}\right)$ for primes $q$ not dividing $N p$. By the Chebotarev density theorem, the set of $\operatorname{Frob}_{q}$ for primes $q$ not dividing $N p$ is dense in $G_{\mathbb{Q}, M_{\bar{\rho}} p}$. Since $\tilde{\tau}^{\Gamma\left(M_{\bar{\rho}}\right)}$ 
is continuous and $\operatorname{Im}(r)$ is closed, it contains $\tilde{\tau}^{\Gamma\left(M_{\bar{\rho}}\right)}(g)$ for every $g \in G_{\mathbb{Q}, M_{\bar{\rho}} p}$. It also contains $q S_{q}=\tilde{\delta}^{\Gamma\left(M_{\bar{\rho}}\right)}\left(\operatorname{Frob}_{q}\right)$ for all the primes $q$ not dividing $N p$. As $\tilde{\delta}^{\Gamma\left(M_{\bar{\rho}}\right)}$ is continuous and $\operatorname{Im}(r)$ is closed, by the Chebotarev density theorem, we see that it contains $\tilde{\delta}^{\Gamma\left(M_{\bar{\rho}}\right)}(g)$ for every $g \in G_{\mathbb{Q}, M_{\bar{\rho}} p}$. For every prime $q$ not dividing $M_{\bar{\rho}}$, we have $\tilde{\tau}^{\Gamma\left(M_{\bar{\rho}}\right)}\left(\operatorname{Frob}_{q}\right)=T_{q}$ and $\tilde{\delta}^{\Gamma\left(M_{\bar{\rho}}\right)}\left(\right.$ Frob $\left._{q}\right)=q S_{q}$. Hence, we see that $\operatorname{Im}(r)$ contains $T_{q}, q S_{q}$ for all the primes $q$ not dividing $M_{\bar{\rho}} p$. Thus, $\operatorname{Im}(r)$ is a closed sub-algebra of $A_{\bar{\rho}}^{\Gamma\left(M_{\bar{\rho}}\right)}$ containing all of its generators. Hence, $\operatorname{Im}(r)=A_{\bar{\rho}}^{\Gamma\left(M_{\bar{\rho}}\right)}$. This proves the lemma.

\section{Proof of Theorem 1}

Throughout this section $\Gamma$ means either $\Gamma_{1}(N)$ or $\Gamma_{0}(N), \Gamma(p)$ means either $\Gamma_{1}(N p)$ or $\Gamma_{0}(N p)$ accordingly and $\Gamma(M)$ means $\Gamma_{1}(M)$ or $\Gamma_{0}(M)$ accordingly. As we noted before, the natural map $\mathbb{T}^{\Gamma, p f} \rightarrow \prod \mathbb{T}_{\bar{\rho}}^{\Gamma, p f}$ is an isomorphism and it lifts the natural map $\mathbb{T}^{\Gamma} \rightarrow$ $\prod \mathbb{T}_{\bar{\rho}}^{\Gamma}$. The corresponding statement for $A^{\Gamma, \mathrm{pf}}$ and $A^{\Gamma}$ is also true. The natural surjective map $\mathbb{T}^{\Gamma, p f} \rightarrow A^{\Gamma, p f}$ sends $\mathbb{T}_{\bar{\rho}}^{\Gamma, p f}$ onto $A_{\bar{\rho}}^{\Gamma, p f}$. The natural surjective map $\mathbb{T}^{\Gamma(p) \text {,full }} \rightarrow A^{\Gamma \text {,full }}$ takes $\mathbb{T}_{\bar{\rho}, \lambda}^{\Gamma(p) \text {,full }}$ onto $A_{\bar{\rho}, \lambda}^{\Gamma, \text { full }}$.

Thus, from Proposition 4 and the discussion above, it follows that $A_{\bar{\rho}, 0}^{\Gamma, \text { full }}$ is isomorphic to $\mathbb{T}_{\bar{\rho}, 0}^{\Gamma(p) \text {,full }} / m_{\Lambda} \mathbb{T}_{\bar{\rho}, 0}^{\Gamma(p), \text { full }}$. By Proposition $5, \mathbb{T}_{\bar{\rho}, 0}^{\Gamma(p) \text {,full }}=\mathbb{T}_{\bar{\rho}}^{\Gamma, \mathrm{pf}}\left[\left[U_{p}\right]\right]$ and $A_{\bar{\rho}, 0}^{\Gamma, \text { full }}=A_{\bar{\rho}}^{\Gamma}\left[\left[U_{p}\right]\right]$. Thus, $A_{\bar{\rho}}^{\Gamma, p f}$ is isomorphic to $\mathbb{T}_{\bar{\rho}}^{\Gamma, p f} / m_{\Lambda} \mathbb{T}_{\bar{\rho}}^{\Gamma, p f}$. As the ideal $m_{\Lambda}$ is generated by two elements, the hauptidealsatz implies that $\operatorname{dim} A_{\bar{\rho}}^{\Gamma, p f} \geq \operatorname{dim} \mathbb{T}_{\bar{\rho}}^{\Gamma(p), \mathrm{pf}}-2$.

Suppose $\bar{\rho}$ is a new $\Gamma$-modular representation. Then, we have proved so far that $\mathbb{T}_{\bar{\rho}}^{\Gamma, p f}$ is a finite extension of $\mathbb{T}_{\bar{\rho}}^{\Gamma}$. Observe that under the surjective map $\mathbb{T}_{\bar{\rho}}^{\Gamma, p f} \rightarrow A_{\bar{\rho}}^{\Gamma, p f}, \mathbb{T}_{\bar{\rho}}^{\Gamma}$ is mapped onto $A_{\bar{\rho}}^{\Gamma}$. This follows from the fact that the map between the partially full Hecke algebras is obtained by first reducing the partially full Hecke algebra in characteristic 0 modulo $\mathfrak{p}$ to get an action on $D^{\Gamma}(\mathbb{F})_{\bar{\rho}}$ and then restricting it to the subspace $S^{\Gamma}(\mathbb{F})_{\bar{\rho}}$. Hence, we see that $A_{\bar{\rho}}^{\Gamma, p f}$ is finite over $A_{\bar{\rho}}^{\Gamma}$.

Therefore, $\operatorname{dim} A_{\bar{\rho}}^{\Gamma, p f}=\operatorname{dim} A_{\bar{\rho}}^{\Gamma}$ and $\operatorname{dim} \mathbb{T}_{\bar{\rho}}^{\Gamma, p f}=\operatorname{dim} \mathbb{T}_{\bar{\rho}}^{\Gamma}$. By the Gouvêa-Mazur infinite fern argument, we see that $\operatorname{dim} \mathbb{T}_{\bar{\rho}}^{\Gamma} \geq 4$ (see [17, Corollary 2.28] and [20, Theorem 1]). Hence, $\operatorname{dim} A_{\bar{\rho}}^{\Gamma} \geq \operatorname{dim} \mathbb{T}_{\bar{\rho}}^{\Gamma}-2 \geq 2$.

Now suppose that $\bar{\rho}$ is not a new $\Gamma$-modular representation. Thus, as remarked before, there exists a proper divisor $M_{\bar{\rho}}$ of $N$ such that $\bar{\rho}$ is a new $\Gamma\left(M_{\bar{\rho}}\right)$-modular representation (we can take it to be the smallest divisor of $N$ such that the eigenspace corresponding to the system of eigenvalues corresponding to $\bar{\rho}$ is nonzero in $\left.S^{\Gamma_{1}\left(M_{\bar{\rho}}\right)}(\mathbb{F})\right)$. If $M_{\bar{\rho}}=1$, then we know that $\operatorname{dim} A_{\bar{\rho}}^{\Gamma\left(M_{\bar{\rho}}\right)}=2$ by Theorem III of [8]. If $M_{\bar{\rho}}>1$, then since $\bar{\rho}$ is a new $\Gamma\left(M_{\bar{\rho}}\right)$-modular representation, repeating the argument given in the previous paragraph for $A_{\bar{\rho}}^{\Gamma\left(M_{\bar{\rho}}\right)}$ and $\mathbb{T}_{\bar{\rho}}^{\Gamma\left(M_{\bar{\rho}}\right)}$ gives $\operatorname{dim} A_{\bar{\rho}}^{\Gamma\left(M_{\bar{\rho}}\right)} \geq 2$ (we can do this since, all the results till the previous section are valid for any level not divisible by $p$. This condition is satisfied by $M_{\bar{\rho}}$ 
as it divides $N$ which is co-prime to $p$ ). Thus, in any case, we see that $\operatorname{dim} A_{\bar{\rho}}^{\Gamma\left(M_{\bar{\rho}}\right)} \geq 2$. By Lemma 8, we have a surjective map $A_{\bar{\rho}}^{\Gamma} \rightarrow A_{\bar{\rho}}^{\Gamma\left(M_{\bar{\rho}}\right)}$. Hence, $\operatorname{dim} A_{\bar{\rho}}^{\Gamma} \geq 2$ when $\bar{\rho}$ is not a new $\Gamma$-modular representation. Thus, Theorem 1 is proved for all the $\Gamma$-modular representations $\bar{\rho}$.

\section{Proof of Theorem 3}

Theorem 5. (Böckle, Diamond-Flach-Guo, Gouvêa-Mazur, Kisin) Under the hypotheses of Theorem 3, the natural map $R_{\bar{\rho}} \rightarrow \mathbb{T}_{\bar{\rho}}^{\Gamma_{1}(N)}$ is an isomorphism between local rings of dimension 4.

Proof. If $\bar{\rho}$ is a $\Gamma_{1}(N)$-modular representation which also satisfies the hypotheses of Theorem 3, then mimicking the proof of Theorem 18 of [8] gives the result of the theorem. We can mimic the argument since, under all these assumptions, the infinite fern argument of Gouvêa-Mazur, [9, Theorem 2.8, Theorem 3.1 and Theorem 3.9], [14, Theorem 1.1], [15, Theorem 3.6] and [25, Main Theorem], which are the key ingredients of the proof of Theorem 18 of [8], hold. Note that the Hecke algebra appearing in [9, Theorem 3.9] is of a higher level $N^{\prime}$ such that $N \mid N^{\prime}$ and $N$ and $N^{\prime}$ have the same prime factors (see the discussion after [9, Theorem 2.7] for more details). But the hypotheses of Theorem 3, along with [11, Proposition 2], ensure that all eigenforms of level $N^{\prime}$ lifting $\bar{\rho}$ arise from newforms of level dividing $N$ which means that the natural restriction map $\mathbb{T}_{\bar{\rho}}^{\Gamma_{1}\left(N^{\prime}\right)} \rightarrow \mathbb{T}_{\bar{\rho}}^{\Gamma_{1}(N)}$ is an isomorphism.

Lemma 9. Under the hypotheses of Theorem 3, the algebra $\mathbb{T}_{\bar{\rho}}^{\Gamma_{1}(N)}$ is flat over $\Lambda$ for the structure of $\Lambda$-algebra on $\mathbb{T}_{\bar{\rho}}^{\Gamma_{1}(N)}$ defined at the end of section 2.2 .

Proof. The proof is similar to that of Lemma 19 of [8]. But we need to make a few changes. Let $\bar{\rho}$ be a $\Gamma_{1}(N)$-modular representation. If the $p$-primary part of $(\mathbb{Z} / N \mathbb{Z})^{*}$ is $\prod_{i=1}^{i=n} \mathbb{Z} / p^{e_{i}} \mathbb{Z}$, then the universal deformation ring $R_{\operatorname{det} \bar{\rho}}$ of $\operatorname{det} \bar{\rho}$ is $\Lambda\left[Y_{1}, . ., Y_{n}\right] /\left(Y_{1}{ }^{p^{e_{1}}}-\right.$ $\left.1, \cdots, Y_{n}{ }^{e^{e_{n}}}-1\right)$. Thus, if $p$ does not divide $\phi(N)$, then $R_{\operatorname{det} \bar{\rho}}=\Lambda$. Using the same arguments as used in the proof of Lemma 19 of [8], we get that $R_{\bar{\rho}}$ is flat over $R_{\operatorname{det} \bar{\rho}}$. Since $R_{\operatorname{det} \bar{\rho}}$ is finite and free over $\Lambda$, it is also flat over $\Lambda$. So, we get that $R_{\bar{\rho}}$ is flat over $\Lambda$. To verify that the map $\Lambda \rightarrow R_{\bar{\rho}} \rightarrow \mathbb{T}_{\bar{\rho}}^{\Gamma_{1}(N)}$ is the same as the map $\Lambda \rightarrow \mathbb{T}_{\bar{\rho}}^{\Gamma_{1}(N)}$ given at the end of section 2.2 , we first show that it is true for all the primes $q \in 1+p \mathbb{Z}_{p}$ which are $1(\bmod N)$. This is shown in exactly the same way as it is showed for all the primes $q \in 1+p \mathbb{Z}_{p}$ in the proof of Lemma 19 of [8]. Since we are choosing the primes which are 1 $(\bmod N)$, the nebentypus is trivial at those primes and hence, we can use the argument used in the proof of Lemma 19 of [8] without modifications. By Dirichlet's theorem on primes in arithmetic progressions, we know that the set of all such primes is dense in 
$1+p \mathbb{Z}_{p}$. Thus, by continuity, we see that the two maps considered above are the same. Under the hypotheses of Theorem 3, the map $R_{\bar{\rho}} \rightarrow \mathbb{T}_{\bar{\rho}}^{\Gamma_{1}(N)}$ is an isomorphism. Hence, we conclude that $\mathbb{T}_{\bar{\rho}}^{\Gamma_{1}(N)}$ is flat over $\Lambda$ for the $\Lambda$-algebra structure defined on $\mathbb{T}_{\bar{\rho}}^{\Gamma_{1}(N)}$ at the end of section 2.2 .

There is a surjective map $\mathbb{T}_{\bar{\rho}}^{\Gamma_{1}(N)} \rightarrow A_{\bar{\rho}}^{\Gamma_{1}(N)}$ whose kernel contains $m_{\Lambda} \mathbb{T}_{\bar{\rho}}^{\Gamma_{1}(N)}$ by Proposition 4. Hence, we get a surjective map $\mathbb{T}_{\bar{\rho}}^{\Gamma_{1}(N)} / m_{\Lambda} \mathbb{T}_{\bar{\rho}}^{\Gamma_{1}(N)} \rightarrow A_{\bar{\rho}}^{\Gamma_{1}(N)}$. Since $\mathbb{T}_{\bar{\rho}}^{\Gamma_{1}(N)}$ is flat over $\Lambda$ by Lemma 9, it follows, from [16, Theorem 10.10], that the Krull dimension of $\mathbb{T}_{\bar{\rho}}^{\Gamma_{1}(N)} / m_{\Lambda} \mathbb{T}_{\bar{\rho}}^{\Gamma_{1}(N)}$ is equal to $\operatorname{dim} \mathbb{T}_{\bar{\rho}}^{\Gamma_{1}(N)}-2=4-2=2$. Thus, the Krull dimension of $A_{\bar{\rho}}^{\Gamma_{1}(N)}$ is at most 2. But, by Theorem 1, the dimension of $A_{\bar{\rho}}^{\Gamma_{1}(N)}$ is at least 2. Hence, the dimension of $A_{\bar{\rho}}^{\Gamma_{1}(N)}$ is exactly 2 . This concludes the proof of first part of Theorem 3 for $\Gamma_{1}(N)$ case.

Let us assume that $\bar{\rho}$ satisfies the hypothesis of Theorem 3 and is also new. Then we have proved that $\mathbb{T}_{\bar{\rho}}^{\Gamma_{1}(N), \text { pf }}$ is an integral extension of $\mathbb{T}_{\bar{\rho}}^{\Gamma_{1}(N)}$ and $A_{\bar{\rho}}^{\Gamma_{1}(N) \text {,pf }}$ is an integral extension of $A_{\bar{\rho}}^{\Gamma_{1}(N)}$. By Proposition 4, we see that the kernel of the natural map $\mathbb{T}^{\Gamma_{1}(N), \text { pf }} \rightarrow A^{\Gamma_{1}(N), p f}$ is $m_{\Lambda} \mathbb{T}^{\Gamma_{1}(N) \text {,pf }}$. Thus, the kernel of the natural map $\mathbb{T}_{\bar{\rho}}^{\Gamma_{1}(N)} \rightarrow A_{\bar{\rho}}^{\Gamma_{1}(N)}$ is contained in every prime ideal of $\mathbb{T}_{\bar{\rho}}^{\Gamma_{1}(N), \mathrm{pf}}$ containing $m_{\Lambda} \mathbb{T}_{\bar{\rho}}^{\Gamma_{1}(N), \mathrm{pf}}$. Hence, by going-up theorem, the kernel is contained in every prime ideal of $\mathbb{T}_{\bar{\rho}}^{\Gamma_{1}(N)}$ containing $m_{\Lambda} \mathbb{T}_{\bar{\rho}}^{\Gamma_{1}(N)}$. Therefore, the natural surjective map $\left(\mathbb{T}_{\bar{\rho}}^{\Gamma_{1}(N)} / m_{\Lambda} \mathbb{T}_{\bar{\rho}}^{\Gamma_{1}(N)}\right)^{\text {red }} \rightarrow$ $\left(A_{\bar{\rho}}^{\Gamma_{1}(N)}\right)^{\text {red }}$ is an isomorphism. Under the hypothesis of Theorem 3 , the surjective map $R_{\bar{\rho}} \rightarrow \mathbb{T}_{\bar{\rho}}^{\Gamma_{1}(N)}$ is an isomorphism. Thus, from the proof of Lemma 9 , it follows that $\left(\tilde{R}_{\bar{\rho}}^{0}\right)^{\text {red }}$ is isomorphic to $\left(R_{\bar{\rho}} / m_{\Lambda} R_{\bar{\rho}}\right)^{\text {red }}$ (this follows from the fact that the universal deformation ring of $\operatorname{det} \bar{\rho}$ is $R_{\operatorname{det} \bar{\rho}}=\Lambda\left[Y_{1}, . ., Y_{n}\right] /\left(Y_{1}{ }^{p^{e_{1}}}-1, \ldots, Y_{n}{ }^{p^{e_{n}}}-1\right)$ and hence, its maximal ideal is $\left.\operatorname{Rad}\left(m_{\Lambda}\right)\right)$ and the map $R_{\bar{\rho}} \rightarrow \mathbb{T}_{\bar{\rho}}^{\Gamma_{1}(N)}$ considered above induces an isomorphism $\left(\tilde{R}_{\bar{\rho}}^{0}\right)^{\text {red }} \rightarrow\left(\mathbb{T}_{\bar{\rho}}^{\Gamma_{1}(N)} / m_{\Lambda} \mathbb{T}_{\bar{\rho}}^{\Gamma_{1}(N)}\right)^{\text {red }}$. As seen before, we have a surjective map $\left(\tilde{R}_{\bar{\rho}}^{0}\right)^{\text {red }} \rightarrow\left(A_{\bar{\rho}}^{\Gamma_{1}(N)}\right)^{\text {red }}$ and this map factors through $\left(\mathbb{T}_{\bar{\rho}}^{\Gamma_{1}(N)} / m_{\Lambda} \mathbb{T}_{\bar{\rho}}^{\Gamma_{1}(N)}\right)^{\text {red }}$. Since both the maps $\left(\tilde{R}_{\bar{\rho}}^{0}\right)^{\text {red }} \rightarrow\left(\mathbb{T}_{\bar{\rho}}^{\Gamma_{1}(N)} / m_{\Lambda} \mathbb{T}_{\bar{\rho}}^{\Gamma_{1}(N)}\right)^{\text {red }}$ and $\left(\mathbb{T}_{\bar{\rho}}^{\Gamma_{1}(N)} / m_{\Lambda} \mathbb{T}_{\bar{\rho}}^{\Gamma_{1}(N)}\right)^{\text {red }} \rightarrow\left(A_{\bar{\rho}}^{\Gamma_{1}(N)}\right)^{\text {red }}$ are isomorphisms, we see that $\left(\tilde{R}_{\bar{\rho}}^{0}\right)^{\text {red }}$ is isomorphic to $\left(A_{\bar{\rho}}^{\Gamma_{1}(N)}\right)^{\text {red }}$ under the map mentioned above.

Now suppose $\bar{\rho}$ satisfies the hypotheses of Theorem 3 but it is not new. Note that, while proving the theorem for a new $\bar{\rho}$ of level $N$, we use the result that $\mathbb{T}_{\bar{\rho}}^{\Gamma_{1}(N) \text {,pf }}$ is an integral extension of $\mathbb{T}_{\bar{\rho}}^{\Gamma_{1}(N)}$ to prove that the kernel of the surjective map $\mathbb{T}_{\bar{\rho}}^{\Gamma_{1}(N)} \rightarrow$ $A_{\bar{\rho}}^{\Gamma_{1}(N)}$ is nilpotent modulo $m_{\Lambda} \mathbb{T}_{\bar{\rho}}^{\Gamma_{1}(N)}$, which in turn implies the isomorphism between $\left(\mathbb{T}_{\bar{\rho}}^{\Gamma_{1}(N)} / m_{\Lambda} \mathbb{T}_{\bar{\rho}}^{\Gamma_{1}(N)}\right)^{\text {red }}$ and $\left(A_{\bar{\rho}}^{\Gamma_{1}(N)}\right)^{\text {red }}$. This, along with the isomorphism between $R_{\bar{\rho}}$ and $\mathbb{T}_{\bar{\rho}}^{\Gamma_{1}(N)}$, gives us the isomorphism between $\left(\tilde{R}_{\bar{\rho}}^{0}\right)^{\text {red }}$ and $\left(A_{\bar{\rho}}^{\Gamma_{1}(N)}\right)^{\text {red }}$. In the present situation, we know that $R_{\bar{\rho}}$ and $\mathbb{T}_{\bar{\rho}}^{\Gamma_{1}(N)}$ are isomorphic. So, to prove the theorem in the current case, it suffices to prove that $\mathbb{T}_{\bar{\rho}}^{\Gamma_{1}(N), \text { pf }}$ is an integral extension of $\mathbb{T}_{\bar{\rho}}^{\Gamma_{1}(N)}$. To prove 
this, we will first compare the Artin conductor of $\bar{\rho}$ with the level $N$ and then do a case by case analysis using the technique used in the proof of Proposition 6. Let $C$ be the Artin conductor of $\bar{\rho}$. Recall that we assume $\bar{\rho}$ to be semi-simple. As there exists a newform of level $N$ giving rise to $\bar{\rho}$, we see that $N=C \prod r^{v(r)}$, where the product is over finitely many primes $r$ and $v(r) \leq 2$ for every $r$ (See [11, Proposition 2] and the discussion before it). Note that the product may include some divisors of $C$.

Let $\ell$ be a prime dividing $N$. So, if $f$ is an eigenform of level $N$ lifting the system of eigenvalues associated to $\bar{\rho}$, then $f$ comes from a newform $g$ of level $M$ such that the highest power of $\ell$ dividing $\frac{N}{M}$ is at most 2. Thus, $f(z)=\sum_{i=1}^{i=r} g\left(d_{i} z\right)$, where $d_{i}$ 's are some divisors of $\frac{N}{M}$. Now we will analyze the action of $U_{\ell}$ on $f$ case by case to find a monic polynomial with coefficients in $\mathbb{T}_{\bar{\rho}}^{\Gamma_{1}(N)}$ satisfied by $U_{\ell}$.

Case 1: $\ell \mid M$ but $\ell \nmid \frac{N}{M}$ : In this case, $\ell \nmid d_{i}$ for all $i$ and hence, $f$ is new at $\ell$. Therefore, from the proof of Proposition 6, we see that $U_{\ell} Q_{\ell}\left(U_{\ell}\right)(f)=0$ where $Q_{\ell}(x)$ is the characteristic polynomial of a lift of $\mathrm{Frob}_{\ell}$ in $G_{\mathbb{Q}, N p}$ as considered in the proof of Proposition 6. Case 2: $\ell \mid M$ and $\ell \| \frac{N}{M}$ : In this case, for every $i$ either $\ell \| d_{i}$ or $\ell \nmid d_{i}$ and $g$ is an eigenform for $U_{\ell}$. So, $U_{\ell} f$ is an eigenform of level $\frac{N}{\ell}$ which is also new at $\ell$ if it is non-zero. So, by the same logic as in the previous case, $U_{\ell} Q_{\ell}\left(U_{\ell}\right)\left(U_{\ell} f\right)=0$.

Case 3: $\ell \mid M$ and $\ell^{2} \mid \frac{N}{M}$ : In this case, for every $i$ either $\ell \nmid d_{i}$ or $\ell$ divides $d_{i}$ with multiplicity at most 2 and $g$ is an eigenform for $U_{\ell}$. So, $U_{\ell}^{2} f$ is an eigenform of level $\frac{N}{\ell^{2}}$ and it is new at $\ell$ when it is non-zero. Thus, we get $U_{\ell} Q_{\ell}\left(U_{\ell}\right)\left(U_{\ell}^{2} f\right)=0$.

Case 4: $\ell \nmid M$ and $\ell \nmid d_{i}$ for all $i$ : If $\ell \nmid M$ and $\ell \nmid d$, then it follows directly from the description of the action of $T_{\ell}$ and $U_{\ell}$ on the $q$-expansions of modular forms, along with the assumption that $g$ is an eigenform of level $M$, that $U_{\ell}$ stabilizes the subspace of modular forms of level $M d \ell$ generated by $g(d z)$ and $g(\ell d z)$ (see [5, Lemma III.7.2] and the discussion around it). Moreover, the characteristic polynomial of $U_{\ell}$ over this subspace is $\left(U_{\ell}^{2}-\operatorname{tr} \rho_{g}\left(\right.\right.$ Frob $\left._{\ell}\right) U_{\ell}+\operatorname{det} \rho_{g}\left(\right.$ Frob $\left.\left._{\ell}\right)\right)$, where $\rho_{g}$ is the $p$-adic Galois representation of $G_{\mathbb{Q}, M p}$ attached to $g$. Thus, in this case, we get that $Q_{\ell}\left(U_{\ell}\right)(f)=0$.

Case 5: $\ell \nmid M$ and $\ell$ divides at least one of the $d_{i}$ 's: Note that, $U_{\ell} g\left(d_{i} z\right)$ is an eigenform of level $\frac{N}{\ell}$ if $\ell \| d_{i}$ and $U_{\ell}^{2} g\left(d_{i} z\right)$ is an eigenform of level $\frac{N}{\ell^{2}}$ if $\ell^{2} \mid d_{i}$. This means that for all $i$, one of the $g\left(d_{i} z\right), U_{\ell} g\left(d_{i} z\right)$ and $U_{\ell}^{2} g\left(d_{i} z\right)$ is an eigenform of level co-prime to $\ell$. So, by case 4 , we have $Q_{\ell}\left(U_{\ell}\right)\left(U_{\ell}^{2} g\left(d_{i} z\right)\right)=0$ for all $i$ as both $Q_{\ell}\left(U_{\ell}\right)\left(U_{\ell} g\left(d_{i} z\right)\right)=0$ and $Q_{\ell}\left(U_{\ell}\right)\left(g\left(d_{i} z\right)\right)=0$ imply $Q_{\ell}\left(U_{\ell}\right)\left(U_{\ell}^{2} g\left(d_{i} z\right)\right)=0$. Hence, we have $Q_{\ell}\left(U_{\ell}\right)\left(U_{\ell}^{2} f\right)=0$.

From the previous paragraphs, we conclude that, if $f$ is an eigenform of level $N$ lifting $\bar{\rho}$, then $\left(U_{\ell}^{3} Q_{\ell}\left(U_{\ell}\right)\right) f=0$ which means $U_{\ell}^{3} Q_{\ell}\left(U_{\ell}\right)=0$. As $Q_{\ell}(x)$ is a monic polynomial with coefficients in $\mathbb{T}_{\bar{\rho}}^{\Gamma_{1}(N)}, U_{\ell}$ is integral over $\mathbb{T}_{\bar{\rho}}^{\Gamma_{1}(N)}$ for all primes $\ell$ dividing $N$. Therefore, $\mathbb{T}_{\bar{\rho}}^{\Gamma_{1}(N), \text { pf }}$ is an integral extension of $\mathbb{T}_{\bar{\rho}}^{\Gamma_{1}(N)}$ and $A_{\bar{\rho}}^{\Gamma_{1}(N), \text { pf }}$ is an integral extension of $A_{\bar{\rho}}^{\Gamma_{1}(N)}$. 
Now using the argument used for the new case above, we get that $\left(\tilde{R}_{\bar{\rho}}^{0}\right)^{\text {red }}$ is isomorphic to $\left(A_{\bar{\rho}}^{\Gamma_{1}(N)}\right)^{\text {red }}$. This concludes the proof of the second part of Theorem 3 for $\Gamma_{1}(N)$ case.

Let $\bar{\rho}$ be a $\Gamma_{0}(N)$-modular representation which satisfies hypothesis of Theorem 3. Then, by the arguments above, it follows that $A_{\bar{\rho}}^{\Gamma_{1}(N)}$ has Krull dimension 2 and $\left(A_{\bar{\rho}}^{\Gamma_{1}(N)}\right)^{\text {red }}$ is isomorphic to $\left(\tilde{R}_{\bar{\rho}}^{0}\right)^{\text {red }}$. We have a natural surjective map $A_{\bar{\rho}}^{\Gamma_{1}(N)} \rightarrow A_{\bar{\rho}}^{\Gamma_{0}(N)}$. By Theorem 1 , the Krull dimension of $A_{\bar{\rho}}^{\Gamma_{0}(N)}$ is at least 2 . Hence, we conclude that the Krull dimension of $A_{\bar{\rho}}^{\Gamma_{0}(N)}$ is exactly 2. If $p$ does not divide $\phi(N)$, then as observed in the introduction, the natural surjective map $A_{\bar{\rho}}^{\Gamma_{1}(N)} \rightarrow A_{\bar{\rho}}^{\Gamma_{0}(N)}$ is an isomorphism. Hence, we get that $\left(A_{\bar{\rho}}^{\Gamma_{0}(N)}\right)^{\text {red }}$ is isomorphic to $\left(\tilde{R}_{\bar{\rho}}^{0}\right)^{\text {red }}$. Thus, Theorem 3 is proved in the $\Gamma_{0}(N)$ case.

Remark. Let $\bar{\rho}$ be a $\Gamma_{1}(N)$-modular representation satisfying the hypothesis of Theorem 3 and assume $p \nmid \phi(N)$. Moreover, assume if $\ell \| N$ and $p \mid \ell+1$, then $\operatorname{det} \bar{\rho}\left(I_{\ell}\right) \neq 1$. Hence, from the proof of Proposition 6 and the proof of Theorem 1, we see that the kernel of the map $\mathbb{T}_{\bar{\rho}}^{\Gamma_{1}(N)} \rightarrow A_{\bar{\rho}}^{\Gamma_{1}(N)}$ is $m_{\Lambda} \mathbb{T}_{\bar{\rho}}^{\Gamma_{1}(N)}$. As $p \nmid \phi(N)$, the kernel of the surjective map $R_{\bar{\rho}} \rightarrow \tilde{R}_{\bar{\rho}}^{0}$ is $m_{\Lambda} R_{\bar{\rho}}$. As $\bar{\rho}$ satisfies the hypotheses of Theorem 3 , we get that $R_{\bar{\rho}} \simeq \mathbb{T}_{\bar{\rho}}^{\Gamma_{1}(N)}$. Hence, by combining all the observations above, we see that the natural surjective map $\tilde{R}_{\bar{\rho}}^{0} \rightarrow A_{\bar{\rho}}^{\Gamma_{1}(N)}$ is an isomorphism. Thus, for this case, we get a stronger statement than Theorem 3.

\section{Proof of Theorem 2}

Throughout this section $\Gamma$ means either $\Gamma_{0}(N)$ or $\Gamma_{1}(N)$ with $p \nmid \phi(N)$. Assume that $\bar{\rho}$ is an unobstructed $\Gamma$-modular representation. So, by assumption, the cotangent space of $\tilde{R}_{\bar{\rho}}^{0}$ has dimension 2 and this implies that its Krull dimension is at most 2 . We have a surjective morphism $\tilde{R}_{\bar{\rho}}^{0} \rightarrow A_{\bar{\rho}}^{\Gamma}$. The Krull dimension of $A_{\bar{\rho}}^{\Gamma}$ is at least 2 by Theorem 1 . Hence, the Krull dimension of $\tilde{R}_{\bar{\rho}}^{0}$ is exactly 2 and it is a regular local ring of dimension 2. Therefore, by [16, Proposition 10.16], it is isomorphic to $\mathbb{F}[[x, y]]$. Hence, the surjective map $\tilde{R}_{\bar{\rho}}^{0} \rightarrow A_{\bar{\rho}}^{\Gamma}$ is an isomorphism. This proves Theorem 2 for the cases considered above.

If $\bar{\rho}$ is an unobstructed $\Gamma_{1}(N)$-modular representation and $p \mid \phi(N)$, then we have a surjective morphism $\tilde{R}_{\bar{\rho}}^{0} \rightarrow\left(A_{\bar{\rho}}^{\Gamma_{1}(N)}\right)^{\text {red }}$. By Theorem 1, $A_{\bar{\rho}}^{\Gamma_{1}(N)}$ and hence, $\left(A_{\bar{\rho}}^{\Gamma_{1}(N)}\right)^{\text {red }}$ both have Krull dimension at least 2. Therefore, the argument used in the previous paragraph proves Theorem 2 in this case as well. This completes the proof of Theorem 2 in all the cases. 


\section{UnOBSTRUCTED MODULAR REPRESENTATIONS}

In this section, we study $\Gamma_{1}(N)$-modular representations to determine if and when they can be unobstructed. If $\bar{\rho}$ is irreducible, then $\bar{\rho}$ is unobstructed in our sense if and only if $H^{1}\left(G_{\mathbb{Q}, N p}, \operatorname{ad}^{0} \bar{\rho}\right)$ has dimension 2 (see section 1.4 of [8]). By Tate's global Euler characteristic formula, we see that $\operatorname{dim} H^{1}\left(G_{\mathbb{Q}, N p}, \operatorname{ad}^{0} \bar{\rho}\right) \geq 2$ with equality if and only if $H^{2}\left(G_{\mathbb{Q}, N p}, \operatorname{ad}^{0} \bar{\rho}\right)=0$. If $p$ does not divide $\phi(N)$, then this is equivalent to $H^{2}\left(G_{\mathbb{Q}, N p}, \operatorname{ad} \bar{\rho}\right)=0$, as $\operatorname{ad} \bar{\rho}=1 \oplus \operatorname{ad}^{0} \bar{\rho}$ and, by the global Euler characteristic formula, $H^{2}\left(G_{\mathbb{Q}, N p}, 1\right)=0$. Thus, in this case, $\bar{\rho}$ is unobstructed in our sense if and only if it is unobstructed in the sense of Mazur ([26], section 1.6). The study of such unobstructed representations is carried out in [31], [32] when $p$ does not divide $\phi(N)$.

However, if $\bar{\rho}$ is irreducible and $p$ divides $\phi(N)$, then the global Euler characteristic formula tells us that $H^{2}\left(G_{\mathbb{Q}, N p}, 1\right) \neq 0$ and hence, $H^{2}\left(G_{\mathbb{Q}, N p}, \operatorname{ad} \bar{\rho}\right) \neq 0$. Thus, we see that $\bar{\rho}$ is always obstructed in the sense of Mazur if $p \mid \phi(N)$ (see Theorem 4.5 of [31]). However, it is not clear a priori if $\bar{\rho}$ can be unobstructed in our sense when $p \mid \phi(N)$. We devote most of this section to study the unobstructedness of reducible and irreducible $\bar{\rho}$ 's when $p \mid \phi(N)$.

Throughout this section, we assume that $p \mid \phi(N)$ unless otherwise stated. Note that, as $p \nmid N$ and $p \mid \phi(N)$, there exists at least one prime divisor $\ell$ of $N$ such that $p \mid \ell-1$ i.e. $\ell \equiv 1(\bmod p)$. Let $\mathcal{C}$ be the category of local profinite $\mathbb{F}$-algebras. Let $D_{\bar{\rho}}$ be the functor from $\mathcal{C}$ to $\mathcal{S E T S}$ of deformations of the pseudo-representation $(\operatorname{tr} \bar{\rho}, \operatorname{det} \bar{\rho})$ and $D_{\bar{\rho}}^{0}$ be its subfunctor of deformations with constant determinant.

Proposition 7. If $p \mid \phi(N)$, then every reducible $\Gamma_{1}(N)$-modular representation is obstructed.

Proof. Let $\bar{\rho}$ be a reducible $\Gamma_{1}(N)$-modular representation. Up to a twist, $\bar{\rho}$ is of the form $1 \oplus \chi$ where $\chi$ is an odd character of $G_{\mathbb{Q}, N p}$ taking values in $\mathbb{F}$. The character $\chi$ is odd because $\bar{\rho}$ is odd. By main theorem of [4], we have the following exact sequence involving tangent space of $D_{\bar{\rho}}$ :

$$
0 \rightarrow \operatorname{Tan}\left(D_{1} \oplus D_{\chi}\right) \stackrel{i}{\rightarrow} \operatorname{Tan}\left(D_{\bar{\rho}}\right) \rightarrow H^{1}\left(G_{\mathbb{Q}, N p}, \chi\right) \otimes H^{1}\left(G_{\mathbb{Q}, N p}, \chi^{-1}\right) \rightarrow H^{2}\left(G_{\mathbb{Q}, N p}, 1\right)^{2}
$$

Here, $D_{\chi}$ and $D_{1}$ are the deformation functors of $\chi$ and 1 as characters of $G_{\mathbb{Q}, N p}$, respectively and $i$ is the map which sends a pair of deformations $(\alpha, \beta)$ of $(\chi, 1)$ on $\mathbb{F}[\epsilon] /\left(\epsilon^{2}\right)$ to the deformation $\alpha+\beta$ of $\operatorname{tr} \bar{\rho}=1+\chi$.

Let $\gamma$ be an element of $H^{1}\left(G_{\mathbb{Q}, N p}, \chi\right) \otimes H^{1}\left(G_{\mathbb{Q}, N p}, \chi^{-1}\right)$ which is the image of some deformation $(t, d)$ of $(\operatorname{tr} \bar{\rho}, \operatorname{det} \bar{\rho})$ to $\mathbb{F}[\epsilon] /\left(\epsilon^{2}\right)$. Thus, $d$ is a deformation of $\operatorname{det} \bar{\rho}=\chi$ and hence, an element of $\operatorname{Tan}\left(D_{\chi}\right) \subset \operatorname{Tan}\left(D_{1} \oplus D_{\chi}\right)$. Thus, subtracting the image of this 
element under the map $i$ from $(t, d)$ gives us an element of $\operatorname{Tan}\left(D_{\bar{\rho}}^{0}\right)$ whose image in $H^{1}\left(G_{\mathbb{Q}, N p}, \chi\right) \otimes H^{1}\left(G_{\mathbb{Q}, N p}, \chi^{-1}\right)$ is still $\gamma$. Therefore, we have an exact sequence:

$$
0 \rightarrow \operatorname{Tan}\left(\left(D_{1} \oplus D_{\chi}\right)^{0}\right) \stackrel{i}{\rightarrow} \operatorname{Tan}\left(D_{\bar{\rho}}^{0}\right) \rightarrow H^{1}\left(G_{\mathbb{Q}, N p}, \chi\right) \otimes H^{1}\left(G_{\mathbb{Q}, N p}, \chi^{-1}\right) \rightarrow H^{2}\left(G_{\mathbb{Q}, N p}, 1\right)^{2}
$$

Here, $\left(D_{1} \oplus D_{\chi}\right)^{0}$ is the subfunctor of $D_{1} \oplus D_{\chi}$ parameterizing the deformations $(\alpha, \beta)$ of $(1, \chi)$ such that $\alpha \beta$ is constant.

Observe that, $\operatorname{dim} H^{1}\left(G_{\mathbb{Q}, N p}, 1\right)=n+1$, where $n=$ number of prime divisors of $N$ which are $1 \bmod p$. Hence, $\operatorname{dim} \operatorname{Tan}\left(\left(D_{1} \oplus D_{\chi}\right)^{0}\right)=n+1$. Since, $i$ is an injective map, we get $\operatorname{dim} \operatorname{Tan}\left(D_{\bar{\rho}}^{0}\right) \geq n+1$. Thus, if $n \geq 2$, then $\operatorname{dim} \operatorname{Tan}\left(D_{\bar{\rho}}^{0}\right) \geq 3$. Hence, $\bar{\rho}$ is obstructed when $n \geq 2$ i.e. when there are at least two primes dividing $N$ which are $1 \bmod p$.

Let us now assume that $n=1$. Thus, there is a unique prime $\ell$ which divides $N$ and which is $1 \bmod p$. In this case, $\operatorname{dim} H^{1}\left(G_{\mathbb{Q}, N p}, 1\right)=2$ and by Tate's global Euler characteristic formula, $\operatorname{dim} H^{2}\left(G_{\mathbb{Q}, N p}, 1\right)=1$. From the exact sequence above, we get that $\operatorname{dim} \operatorname{Tan}\left(D_{\bar{\rho}}^{0}\right) \geq 2+\operatorname{dim} H^{1}\left(G_{\mathbb{Q}, N p}, \chi\right) \operatorname{dim} H^{1}\left(G_{\mathbb{Q}, N p}, \chi^{-1}\right)-2 \operatorname{dim} H^{2}\left(G_{\mathbb{Q}, N p}, 1\right)$.

We shall distinguish between two cases:

First case: $\left.\chi\right|_{G_{\mathbb{Q}_{\ell}}}=1$

Note that, as $\ell \equiv 1(\bmod p),\left.\omega_{p}\right|_{G_{\mathbb{Q} \ell}}=1$.

If $\chi \neq \omega_{p}$, then, by Greenberg-Wiles version of Poitou-Tate duality ([30, Theorem 2]), we get that $\operatorname{dim} H^{1}\left(G_{\mathbb{Q}, N p}, \chi\right) \geq \operatorname{dim} H^{1}\left(G_{\mathbb{Q}_{\ell}},\left.\chi\right|_{G_{\mathbb{Q}_{\ell}}}\right)-\operatorname{dim} H^{0}\left(G_{\mathbb{Q}_{\ell}},\left.\chi\right|_{G_{\mathbb{Q}_{\ell}}}\right)+\operatorname{dim} H^{1}\left(G_{\mathbb{Q}_{p}},\left.\chi\right|_{G_{\mathbb{Q}_{p}}}\right)-$ $\operatorname{dim} H^{0}\left(G_{\mathbb{Q}_{p}},\left.\chi\right|_{G_{\mathbb{Q}_{p}}}\right)$. As $\ell \equiv 1(\bmod p), \operatorname{dim} H^{1}\left(G_{\mathbb{Q}_{\ell}}, 1\right)=2$. By the local Euler characteristic formula, $\operatorname{dim} H^{1}\left(G_{\mathbb{Q}_{p}},\left.\chi\right|_{G_{\mathbb{Q}_{p}}}\right)-\operatorname{dim} H^{0}\left(G_{\mathbb{Q}_{p}},\left.\chi\right|_{G_{\mathbb{Q}_{p}}}\right)=1+\operatorname{dim} H^{2}\left(G_{\mathbb{Q}_{p}},\left.\chi\right|_{G_{\mathbb{Q}_{p}}}\right) \geq 1$. Since, $\left.\chi\right|_{G_{\mathbb{Q}_{\ell}}}=1$, we see that $\operatorname{dim} H^{1}\left(G_{\mathbb{Q}, N p}, \chi\right) \geq 2-1+1=2$.

If $\chi=\omega_{p}$, then, using the Kümmer exact sequence, we get that a class in $H^{1}\left(G_{\mathbb{Q}, N p}, \omega_{p}\right)$ is represented by a co-cycle of the form $g \mapsto g(\alpha) / \alpha$, with $\alpha \in \overline{\mathbb{Q}}, \alpha^{p} \in \mathbb{Q}$ and $v_{q}\left(\alpha^{p}\right)=0$ for all primes $q \nmid N p$ (See section 1 of [30] for more details). Hence, $\operatorname{dim} H^{1}\left(G_{\mathbb{Q}, N p}, \omega_{p}\right)=$ $n^{\prime}$, where $n^{\prime}=$ number of distinct prime factors of $N p$. Hence, $\operatorname{dim} H^{1}\left(G_{\mathbb{Q}, N p}, \omega_{p}\right) \geq 2$. Since, $\left.\chi^{-1}\right|_{G_{\mathbb{Q}_{\ell}}}=1$ as well, the same argument gives us $\operatorname{dim} H^{1}\left(G_{\mathbb{Q}, N p}, \chi^{-1}\right) \geq 2$.

Thus, combining all the calculations above, we get that $\operatorname{dim} \operatorname{Tan}\left(D_{\bar{\rho}}^{0}\right) \geq 2+(2)(2)-$ $2(1)=4$. Hence, if $n=1$ and $\left.\chi\right|_{G_{\mathbb{Q}_{\ell}}}=1$, then $\bar{\rho}$ is obstructed.

Second case: $\left.\chi\right|_{G_{Q_{\ell}}} \neq 1$

As $\left.\chi\right|_{G_{\mathbb{Q}_{\ell}}} \neq 1, \omega_{p} \otimes \chi \neq 1$. Thus, $H^{0}\left(G_{\mathbb{Q}_{\ell}},\left.\chi\right|_{G_{\mathbb{Q}_{\ell}}}\right)=0, H^{2}\left(G_{\mathbb{Q}_{\ell}},\left.\chi\right|_{G_{\mathbb{Q}_{\ell}}}\right)=H^{0}\left(G_{\mathbb{Q}_{\ell}}, \omega_{p} \otimes\right.$ $\left.\left.\chi\right|_{G_{\mathbb{Q}_{\ell}}}\right)=0$ and hence, $H^{1}\left(G_{\mathbb{Q}_{\ell}},\left.\chi\right|_{G_{\mathbb{Q}_{\ell}}}\right)=0$. Similarly, we get that $H^{1}\left(G_{\mathbb{Q}_{\ell}},\left.\chi^{-1}\right|_{G_{\mathbb{Q}_{\ell}}}\right)=0$. Thus, the calculations done in the first case do not imply that $\operatorname{dim} \operatorname{Tan}\left(D_{\bar{\rho}}^{0}\right)>2$ which is required to prove that $\bar{\rho}$ is obstructed. 
Let us assume that $\bar{\rho}$ is unobstructed. So, the dimension of the tangent space of $\tilde{R}_{\bar{\rho}}^{0}$ is 2 . By Theorem 1, its Krull dimension is at least 2 . Hence, $\tilde{R}_{\bar{\rho}}^{0}$ is isomorphic to $\mathbb{F}[[x, y]]$, the power series ring in two variables. Let $\left(t^{\text {univ }}, d^{\text {univ }}\right)$ be the universal pseudo-representation with constant determinant in characteristic $p$ deforming $(\operatorname{tr} \bar{\rho}, \operatorname{det} \bar{\rho})$.

By [6, Theorem 1.4.4, Chapter 1], $\tilde{R}_{\bar{\rho}}^{0}\left[G_{\mathbb{Q}_{\ell}}\right] /\left(\operatorname{ker}\left(t^{\text {univ }} \circ i_{\ell}\left(G_{\mathbb{Q}_{\ell}}\right)\right)\right.$ is a Generalized Matrix Algebra (GMA) of the form $\left(\begin{array}{cc}\tilde{R}_{\bar{\rho}}^{0} & B \\ C & \tilde{R}_{\bar{\rho}}^{0}\end{array}\right)$, where $B$ and $C$ are finitely generated $\tilde{R}_{\bar{\rho}^{-}}^{0}$ modules and the diagonal entries reduce to 1 and $\chi$ modulo the maximal ideal of $\tilde{R}_{\bar{\rho}}^{0}$. By [6, Theorem 1.5.5, Chapter 1], we get injective maps $\left(B / m_{\tilde{R}_{\bar{\rho}}^{0}} B\right)^{*} \hookrightarrow \operatorname{Ext}_{G_{\mathbb{Q}_{\ell}}}^{1}(\chi, 1)$ and $\left(C / m_{\tilde{R}_{\bar{\rho}}^{0}} C\right)^{*} \hookrightarrow \operatorname{Ext}_{G_{\mathbb{Q}_{\ell}}}^{1}(1, \chi)$. Since, we have $H^{1}\left(G_{\mathbb{Q}_{\ell}},\left.\chi\right|_{G_{\mathbb{Q}_{\ell}}}\right)=H^{1}\left(G_{\mathbb{Q}_{\ell}},\left.\chi^{-1}\right|_{G_{\mathbb{Q}_{\ell}}}\right)=$ 0 , it implies that $\operatorname{Ext}_{G_{\mathbb{Q}_{\ell}}}^{1}(\chi, 1)=\operatorname{Ext}_{G_{\mathbb{Q}_{\ell}}}^{1}(1, \chi)=0$ and hence, $B=C=0$. Thus, $t^{\text {univ }} \circ i_{\ell}\left(G_{\mathbb{Q}_{\ell}}\right)=\kappa_{1}+\kappa_{2}$, where $\kappa_{1}$ and $\kappa_{2}$ are characters of $G_{\mathbb{Q}_{\ell}}$ taking values in $\left(\tilde{R}_{\bar{\rho}}^{0}\right)^{*}$ and are deformations of 1 and $\chi$, respectively. Therefore, $t^{\text {univ }} \circ i_{\ell}$ factors through $G_{\mathbb{Q}_{\ell}}^{\mathrm{ab}}$, the abelianization of $G_{\mathbb{Q}_{\ell}}$.

By local class field theory, $G_{\mathbb{Q}_{\ell}}^{\mathrm{ab}}=\mathbb{Z}_{\ell}^{*} \times \hat{\mathbb{Z}}$. Let $p^{e}$ be the highest power of $p$ dividing $\ell-1$. Let $a \in G_{\mathbb{Q}_{\ell}}^{\mathrm{ab}}$ be the unique element of order $p^{e}$. Since, $a$ has order $p^{e}$ and $\mathbb{F}[[x, y]]$ does not have any nontrivial element of order $p$, it follows that $\kappa_{1}(a)=\kappa_{2}(a)=1$. Let $b$ be a lift of $a$ in $G_{\mathbb{Q}_{\ell}}$. Hence, $\left.t^{\text {univ }} \circ i_{\ell}(b)\right)=2$ i.e. it is constant. Hence, $t \circ i_{\ell}(b)=2$ for any deformation $t$ of $\operatorname{tr} \bar{\rho}$ as a pseudo-character of $G_{\mathbb{Q}, N p}$ with constant determinant in characteristic $p$. But it is easy to construct an explicit such deformation $t$ with $t \circ i_{\ell}(b) \neq 2$.

Indeed, to construct such a deformation, first observe that the maximal pro- $p$ subgroup $G_{\mathbb{Q}, N p}^{\mathrm{ab}, p}$ of $G_{\mathbb{Q}, N p}^{\mathrm{ab}}$ is $\mathbb{Z} / p^{e} \mathbb{Z} \times \mathbb{Z}_{p}$. Consider a deformation $\tilde{1}$ of 1 as a character of $G_{\mathbb{Q}, N p}$ to $R=\mathbb{F}[[x]][y] /\left(y^{p^{e}}-1\right)$ which maps $G_{\mathbb{Q}, N p}^{\mathrm{ab}, p} \rightarrow 1+m_{R}$ in the following way: the topological generator of $\mathbb{Z}_{p}$ is mapped to $1+x$ and the generator of $\mathbb{Z} / p^{e} \mathbb{Z}$ is mapped to $y$. The character $\chi / \tilde{1}$, which we will denote by $\tilde{\chi}$, is a deformation of $\chi$. Let $\tilde{\rho}=\tilde{1} \oplus \tilde{\chi}$. Thus, $(\operatorname{tr} \tilde{\rho}, \operatorname{det} \tilde{\rho})$ is a deformation of $(\operatorname{tr} \bar{\rho}, \operatorname{det} \bar{\rho})$ to $R$ with constant determinant. We claim that $\operatorname{tr}\left(\tilde{\rho} \circ i_{\ell}(b)\right) \neq 2$.

To prove the claim, first consider the map $i_{\ell}^{\mathrm{ab}}: G_{\mathbb{Q}_{\ell}}^{\mathrm{ab}} \rightarrow G_{\mathbb{Q}, N p}^{\mathrm{ab}}$ induced from $i_{\ell}$ by passing to the abelianzations of both the groups. By class field theory, $G_{\mathbb{Q}, N p}^{\mathrm{ab}}=\prod \mathbb{Z}_{q}^{*}$ where the product is taken over primes $q$ which divide $N p$. By the local-global compatibility of class field theory, $\mathbb{Z}_{\ell}^{*}$ component of $G_{\mathbb{Q}, N p}^{\mathrm{ab}}$, lies in the image of $\mathbb{Z}_{\ell}^{*} \subset G_{\mathbb{Q} \ell}^{\mathrm{ab}}$ under the map $i_{\ell}^{\mathrm{ab}}$. Thus, the unique element of $G_{\mathbb{Q}, N p}^{\mathrm{ab}}$ of order $p^{e}$ is $i_{\ell}^{\mathrm{ab}}(a)$. Therefore, $\operatorname{tr}\left(\tilde{\rho} \circ i_{\ell}(b)\right)=$ $\tilde{1}\left(i_{\ell}^{\mathrm{ab}}(a)\right)+\tilde{\chi}\left(i_{\ell}^{\mathrm{ab}}(a)\right)=y+y^{-1}$. If $y+y^{-1}=2$, then it would imply $(y-1)^{2}=0$. But this relation does not hold in $R$. Hence, $\operatorname{tr}\left(\tilde{\rho} \circ i_{\ell}(b)\right) \neq 2$ and our claim is proved.

Thus, we get a contradiction to our hypothesis that $\bar{\rho}$ is unobstructed. Therefore, in this case as well, $\bar{\rho}$ is obstructed. 
Therefore, combining all the results above, we get that if $\bar{\rho}$ is a reducible $\Gamma_{1}(N)$ modular representation and if $p \mid \phi(N)$, then $\bar{\rho}$ is obstructed. This finishes the proof of Proposition 7.

Remark. Now suppose that $\bar{\rho}$ is a reducible $\Gamma_{1}(N)$-modular representation and $p$ does not divide $\phi(N)$. Up to a twist, $\bar{\rho}=1 \oplus \chi$. If $p \nmid \phi(N)$, then, $\operatorname{dim} H^{1}\left(G_{\mathbb{Q}, N p}, 1\right)=1$ and, Tate's global Euler characteristic formula implies that $H^{2}\left(G_{\mathbb{Q}, N p}, 1\right)=0$. The exact sequence of [4] considered above implies $\operatorname{dim} \operatorname{Tan}\left(D_{\bar{\rho}}^{0}\right)=1+\operatorname{dim} H^{1}\left(G_{\mathbb{Q}, N p}, \chi\right) \operatorname{dim} H^{1}\left(G_{\mathbb{Q}, N p}, \chi^{-1}\right)$. If $\chi=\omega_{p}$, then by the Kümmer theory argument above, it follows that $\operatorname{dim} H^{1}\left(G_{\mathbb{Q}, N p}, \omega_{p}\right)$ is the number of distinct prime divisors of $N p$ and hence, greater than 1. Hence, if $\chi=\omega_{p}, \omega_{p}^{-1}$, then $\operatorname{dim} \operatorname{Tan}\left(D_{\bar{\rho}}^{0}\right)>2$. Hence, in this case $\bar{\rho}$ is obstructed. If $\chi \neq$ $\omega_{p}, \omega_{p}{ }^{-1}$, then by Greenberg-Wiles version of Poitou-Tate duality, we get that $k+1 \leq$ $\operatorname{dim} H^{1}\left(G_{\mathbb{Q}, N p}, \chi\right) \leq k+1+\operatorname{dim} A\left(\chi^{-1} \omega_{p}\right)$ and $k^{\prime}+1 \leq \operatorname{dim} H^{1}\left(G_{\mathbb{Q}, N p}, \chi^{-1}\right) \leq k^{\prime}+1+$ $\operatorname{dim} A^{\prime}\left(\chi \omega_{p}\right)$, where $k$ is the number of prime divisors $\ell$ of $N$ such that $\left.\chi\right|_{G_{\mathbb{Q}_{\ell}}}=\left.\omega_{p}\right|_{G_{\mathbb{Q}_{\ell}}}, k^{\prime}$ is the number of prime divisors $\ell$ of $N$ such that $\left.\chi^{-1}\right|_{G_{\mathbb{Q}_{\ell}}}=\left.\omega_{p}\right|_{G_{\mathbb{Q}_{\ell}}}, A\left(\chi^{-1} \omega_{p}\right)$ is the part of the $p$-torsion subgroup of the class group of the totally real abelian extension $F$ of $\mathbb{Q}$ fixed by $\operatorname{Ker}\left(\chi^{-1} \omega_{p}\right)$ on which $\operatorname{Gal}(F / \mathbb{Q})$ acts by $\chi^{-1} \omega_{p}$ and $A^{\prime}\left(\chi \omega_{p}\right)$ is the part of the $p$-torsion subgroup of the class group of the totally real abelian extension $F^{\prime}$ of $\mathbb{Q}$ fixed by $\operatorname{Ker}\left(\chi \omega_{p}\right)$ on which $\operatorname{Gal}\left(F^{\prime} / \mathbb{Q}\right)$ acts by $\chi \omega_{p}$.

Proposition 8. Suppose $p \mid \phi(N)$. Let $\bar{\rho}$ be an absolutely irreducible $\Gamma_{1}(N)$-modular representation such that $\left.\bar{\rho}\right|_{G_{\mathbb{Q}_{\ell}}}$ is reducible for at least one prime $\ell$ dividing $N$ which is 1 mod p. Then, $\bar{\rho}$ is obstructed.

Proof. Let $\bar{\rho}$ be an absolutely irreducible $\Gamma_{1}(N)$-modular representation. By [32, Lemma 2.5], we have:

$$
\operatorname{dim}_{\mathbb{F}} H^{2}\left(G_{\mathbb{Q}, N p}, \operatorname{ad} \bar{\rho}\right)=\operatorname{dim}_{\mathbb{F}} \amalg^{1}\left(G_{\mathbb{Q}, N p}, \omega_{p} \otimes \operatorname{ad} \bar{\rho}\right)+\Sigma_{q \mid N p} \operatorname{dim}_{\mathbb{F}} H^{0}\left(G_{\mathbb{Q}_{q}}, \omega_{p} \otimes \operatorname{ad} \bar{\rho}\right)
$$

Note that $\operatorname{ad} \bar{\rho}=1 \oplus \operatorname{ad}^{0} \bar{\rho}$. By Tate's global Euler characteristic formula, we get that $\operatorname{dim}_{\mathbb{F}} H^{2}\left(G_{\mathbb{Q}, N p}, 1\right)=n$, where $n=$ number of prime divisors of $N$ which are $1 \bmod p$. For a prime $q, \operatorname{dim}_{\mathbb{F}} H^{0}\left(G_{\mathbb{Q}_{q}}, \omega_{p}\right)$ is 1 if $q$ is $1 \bmod p$ and 0 , otherwise. By removing the contributions of the trivial representation from both sides of the formula above, we get $\operatorname{dim}_{\mathbb{F}} H^{2}\left(G_{\mathbb{Q}, N p}, \operatorname{ad}^{0} \bar{\rho}\right)=\operatorname{dim}_{\mathbb{F}} \amalg^{1}\left(G_{\mathbb{Q}, N p}, \omega_{p} \otimes \operatorname{ad}^{0} \bar{\rho}\right)+\Sigma_{q \mid N p} \operatorname{dim}_{\mathbb{F}} H^{0}\left(G_{\mathbb{Q}_{q}}, \omega_{p} \otimes \operatorname{ad}^{0} \bar{\rho}\right)$.

Now, let $\ell$ be a prime dividing $N$ such that $\ell-1$ is divisible by $p$. Suppose that $\left.\bar{\rho}\right|_{G_{\mathbb{Q}_{\ell}}}$ is reducible. Thus, $\left.\bar{\rho}\right|_{G_{\mathbb{Q}_{\ell}}}$ is an extension of a character $\chi_{1}$ by a character $\chi_{2}$. If $\chi_{1} \neq \chi_{2}$, then, by Tate's local Euler characteristic formula, $\operatorname{Ext}_{G_{\mathbb{Q}_{\ell}}}^{1}\left(\chi_{1}, \chi_{2}\right)=0$. So, $\left.\bar{\rho}\right|_{G_{\mathbb{Q}_{\ell}}}=\chi_{1} \oplus \chi_{2}$ and $\left.\operatorname{ad}^{0} \bar{\rho}\right|_{G_{\mathbb{Q}_{\ell}}}=1 \oplus \chi_{1} \chi_{2}{ }^{-1} \oplus \chi_{1}{ }^{-1} \chi_{2}$. As $p \mid \ell-1, \omega_{p}\left(G_{\mathbb{Q}_{\ell}}\right)=1$ and $H^{0}\left(G_{\mathbb{Q}_{\ell}}, \omega_{p} \otimes \operatorname{ad}^{0} \bar{\rho}\right)=H^{0}\left(G_{\mathbb{Q}_{\ell}}, \operatorname{ad}^{0} \bar{\rho}\right)$ and in this case, both of them are nonzero. Hence, if $\chi_{1} \neq \chi_{2}$, then, by the formula above, $H^{2}\left(G_{\mathbb{Q}, N p}, \operatorname{ad}^{0} \bar{\rho}\right) \neq 0$ and $\bar{\rho}$ is obstructed. 
If $\chi_{1}=\chi_{2}=\chi$, then $\left.\rho\right|_{G_{\mathbb{Q}_{\ell}}}$ is either $\chi \oplus \chi$ or a nontrivial extension of $\chi$ by itself. If $\left.\rho\right|_{G_{\mathbb{Q}_{\ell}}}=\chi \oplus \chi$, then clearly $1 \subset \operatorname{ad}^{0} \bar{\rho}$. If $\left.\rho\right|_{G_{\mathbb{Q}_{\ell}}}$ is a non-trivial extension of $\chi$ by itself, then choose a basis of $\bar{\rho}$ such that $\bar{\rho}\left(G_{\mathbb{Q}_{\ell}}\right)$ is upper triangular and identify $\operatorname{ad}^{0} \bar{\rho}$ with the subspace of trace 0 matrices of $M_{2}(\mathbb{F})$. Then, $G_{\mathbb{Q}_{\ell}}$ acts trivially on the subspace of $\operatorname{ad}^{0} \bar{\rho}$ generated by the element $\left(\begin{array}{ll}0 & 1 \\ 0 & 0\end{array}\right)$. Thus, $1 \subset \operatorname{ad}^{0} \bar{\rho}$ in this case also. Therefore, if $\chi_{1}=\chi_{2}$, then $H^{0}\left(G_{\mathbb{Q}_{\ell}}, \omega_{p} \otimes \operatorname{ad}^{0} \bar{\rho}\right)=H^{0}\left(G_{\mathbb{Q}_{\ell}}, \operatorname{ad}^{0} \bar{\rho}\right) \neq 0$ and hence, by the formula above, $H^{2}\left(G_{\mathbb{Q}, N p}, \operatorname{ad}^{0} \bar{\rho}\right) \neq 0$ which implies that $\bar{\rho}$ is obstructed. This concludes the proof of Proposition 8.

We would like to determine the cases when the situation considered as above would arise i.e. when $\left.\bar{\rho}\right|_{G_{\mathbb{Q}_{\ell}}}$ would be reducible. Note that, if $f$ is an eigenform of level $N$ and $\rho_{f}$ is the Galois representation attached to it, then we know all the possible descriptions of $\left.\rho_{f}\right|_{G_{\mathbb{Q}_{\ell}}}$ for a prime $\ell$ dividing $N$. So, we will now analyze all the possible descriptions of $\left.\rho_{f}\right|_{G_{\mathbb{Q}_{\ell}}}$ for an eigenform $f$ lifting $\bar{\rho}$ to determine which of them will make $\bar{\rho}$ obstructed and when can they arise. This will give us some conditions on $\bar{\rho}$ which will force it to be obstructed.

Let $f$ be an eigenform of level $N$ lifting $\bar{\rho}, \ell$ be a prime dividing $N$ which is $1 \bmod p$, $\rho_{f}$ be the $p$-adic Galois representation attached to $f$ and $\pi_{\ell}$ be the $\ell$-component of the automorphic representation associated to $f$. If $\pi_{\ell}$ is either principal series or special, then by the local Langlands correspondence, we see that $\left.\rho_{f}\right|_{G_{\mathbb{Q}_{\ell}}}$ and hence, $\left.\bar{\rho}\right|_{G_{\mathbb{Q}_{\ell}}}$ is reducible (see section 3 and section 5 of [31] for a similar analysis). This implies that $\bar{\rho}$ is obstructed. In particular, if $\ell \mid N$ but $\ell^{2} \nmid N$, then we know that $\pi_{\ell}$ is either special or principal series (see section 1.2 of [11]). If $(\bar{\rho})^{I_{\ell}}$, the subspace of $\bar{\rho}$ on which $I_{\ell}$ acts trivially, is non-zero, then clearly $\left.\bar{\rho}\right|_{G_{\mathbb{Q}_{\ell}}}$ is reducible. If there exists an eigenform $f$ of level $M$ which lifts $\bar{\rho}$ such that there exists at least one prime divisor $\ell$ of $N$ which is $1 \bmod p$ and which does not divide $M$, then $\bar{\rho}$ is unramified at $\ell$ and hence, $\left.\bar{\rho}\right|_{G_{\mathbb{Q}_{\ell}}}$ is reducible. Therefore, in these cases, $\bar{\rho}$ is obstructed.

On the other hand, if $\pi_{\ell}$ is supercuspidal, then, as $\ell \neq 2$, it follows, from the local Langlands correspondence, that $\left.\rho_{f}\right|_{G_{\mathbb{Q}_{\ell}}}$ is induced from a character $\chi$ of $G_{K}=\operatorname{Gal}\left(\overline{\mathbb{Q}_{\ell}} / K\right)$, where $K$ is a quadratic extension of $\mathbb{Q}_{\ell}$ and moreover, $\left.\rho_{f}\right|_{G_{\mathbb{Q}_{\ell}}}$ is irreducible (see the proof of Proposition 3.2 of [31]). Let $\chi^{\sigma}$ be the $\operatorname{Gal}\left(K / \mathbb{Q}_{\ell}\right)$-conjugate character of $\chi$. So, $\left.\rho_{f}\right|_{G_{K}} \simeq \chi \oplus \chi^{\sigma}$. If $\left.\bar{\rho}\right|_{G_{\mathbb{Q}_{\ell}}}$ is reducible, then clearly, $\bar{\chi}=\overline{\chi^{\sigma}}$ where $\bar{\chi}$ and $\overline{\chi^{\sigma}}$ are the reductions of $\chi$ and $\chi^{\sigma}$ in characteristic $p$, respectively. This means that $\frac{\chi}{\chi^{\sigma}}$ factors through the maximal abelian pro- $p$ quotient of $G_{K}$. But it follows, from local class field theory and the assumption that $p \mid \ell-1$, that maximal abelian pro- $p$ extension of $K$ is also abelian over $\mathbb{Q}_{\ell}$. So, if $\frac{\chi}{\chi^{\sigma}}$ factors through the maximal abelian pro- $p$ quotient of $G_{K}$, then it implies that $\left.\rho_{f}\right|_{G_{\mathbb{Q}_{\ell}}}=\operatorname{Ind}_{G_{K}}^{G_{\mathbb{Q}_{\ell}}} \chi$ is reducible, contradicting the hypothesis that 
it is irreducible. Thus, we see that if $\pi_{\ell}$ is supercuspidal and $p \mid \ell-1$, then $\left.\bar{\rho}\right|_{G_{\mathbb{Q}_{\ell}}}$ is not reducible. We would like to record these results in the following corollary:

Corollary 4. Suppose $p \mid \phi(N)$. Let $\bar{\rho}$ be an absolutely irreducible $\Gamma_{1}(N)$-modular representation such that it satisfies one of the following conditions:

(1) There exists at least one prime $\ell$ which is $1 \bmod p$ such that $\ell \mid N$ and $\ell^{2} \nmid N$.

(2) There exists an eigenform $f$ of level $M$ which lifts $\bar{\rho}$ such that there is at least one prime divisor $\ell$ of $N$ which is $1 \bmod p$ and which either does not divide $M$ or divides $M$ with multiplicity 1.

(3) The subspace $(\bar{\rho})^{I_{\ell}}$ is non-trivial.

Then, $\bar{\rho}$ is obstructed.

The only case which remains to be considered is when $\left.\bar{\rho}\right|_{G_{\mathbb{Q}_{\ell}}}$ is irreducible for all the prime divisors $\ell$ of $N$ which are $1 \bmod p$. Let $\bar{\rho}$ be such a $\Gamma_{1}(N)$-modular representation. In this case, $H^{0}\left(G_{\mathbb{Q}_{\ell}}, \omega_{p} \otimes \operatorname{ad}^{0} \bar{\rho}\right)=H^{0}\left(G_{\mathbb{Q}_{\ell}}, \operatorname{ad}^{0} \bar{\rho}\right)=0$ for all such primes $\ell$. Note that, if $f$ is any eigenform which lifts $\bar{\rho}$, then the local component $\pi_{\ell}$ of the automorphic representation associated to $f$ is supercuspidal at all such primes. The analysis of contributions coming from $p$ and other prime divisors of $N$ in the formula above, which are not $1 \bmod p$, is done in sections 3-5 of [31] and section 3 of [32] to give conditions for vanishing of corresponding $\mathrm{H}^{0}$ 's. We can assume that those conditions hold as well so that we can ignore those primes. Note that these conditions neither depend on each other nor put any restrictions on each other. So assuming them together does not yield any immediate contradiction. Now, moreover assume that $\bar{\rho}$ does not come from a weight 1 modular form. We need this hypothesis to use the results of section 3 of [15]. We will analyze the only remaining group $\amalg^{1}\left(G_{\mathbb{Q}, N p}, \omega_{p} \otimes \operatorname{ad}^{0} \bar{\rho}\right)$ in this case following the approach of [31] and [32].

Let $g$ be an eigenform such that $\rho_{g}$ is a minimally ramified lift of $\bar{\rho}$ (such a lift does exist, see section 3.2 of [15]). Hence, it is an eigenform of level $N$ and its weight $k$ lies between 2 and $p-1$. Let $K$ be a finite extension of $\mathbb{Q}_{p}$ generated by the eigenvalues of $g$, $\mathcal{O}$ be its ring of integers and $\mathfrak{m}$ be its maximal ideal. Denote by $A_{\rho_{g}}$ the module $(K / \mathcal{O})^{3}$ on which $G_{\mathbb{Q}, N p}$ acts via $\operatorname{ad}^{0} \rho_{g}$. Following [15] and [32], we define $H_{\emptyset}^{1}\left(G_{\mathbb{Q}}, A_{\rho_{g}}\right)$ in the same way as defined in [15, Section 2.1] (See section 2.2 of [32] also).

By $\left[15\right.$, Theorem 2.15], we get that $H_{f}^{1}\left(G_{\mathbb{Q}}, \rho_{g}\right)=H_{f}^{1}\left(G_{\mathbb{Q}}, \rho_{g}(1)\right)=0$, where $\rho_{g}(1)$ is the Tate twist of $\rho_{g}$ and $H_{f}^{1}\left(G_{\mathbb{Q}}, \rho_{g}\right), H_{f}^{1}\left(G_{\mathbb{Q}}, \rho_{g}(1)\right)$ are the Bloch-Kato Selmer groups defined as in section 2.1 of [15]. It follows, from [32, Lemma 2.6], that $\operatorname{dim}_{\mathbb{F}} \amalg^{1}\left(G_{\mathbb{Q}, N p}, \omega_{p} \otimes\right.$ $\left.\operatorname{ad}^{0} \bar{\rho}\right) \leq \operatorname{dim}_{\mathbb{F}} H_{\emptyset}^{1}\left(G_{\mathbb{Q}}, A_{\rho_{g}}\right)[\mathfrak{m}]\left(\mathfrak{m}\right.$ torsion of $\left.H_{\emptyset}^{1}\left(G_{\mathbb{Q}}, A_{\rho_{g}}\right)\right)$. Now, [15, Theorem 3.7] implies that the length of $H_{\emptyset}^{1}\left(G_{\mathbb{Q}}, A_{\rho_{g}}\right)$ is $v_{\mathfrak{m}}\left(\eta_{g}^{\emptyset}\right)$. Here, $v_{\mathfrak{m}}$ is the $\mathfrak{m}$-adic valuation and $\eta_{g} \emptyset$ is the 
congruence ideal of $g$ defined in [15, Section 1.7]. From the proof of [32, Proposition 4.2], we see that $v_{\mathfrak{m}}\left(\eta_{g}{ }^{\emptyset}\right)>0$ if and only if $\mathfrak{m}$ is a congruence prime for $g$ i.e. if there exists an eigenform $h$ which lifts $\bar{\rho}$ and which is not a Galois conjugate of $g$.

Let $\epsilon$ be the nebentypus of $g$. Let $\psi$ be a character of $(\mathbb{Z} / N \mathbb{Z})^{*}$ whose order is a power of $p$ (there exists such a character as $p \mid \phi(N))$. Thus, $\epsilon$ and $\psi \epsilon$ have the same reduction modulo $\mathfrak{m}$. Hence, from [11, Proposition 3], it follows that there exists an eigenform $h$ of level $N$ and nebentypus $\psi \epsilon$ which lifts $\bar{\rho}$. If $h$ is a Galois conjugate of $g$, then $\epsilon\left((\mathbb{Z} / N \mathbb{Z})^{*}\right)$ and $\psi \epsilon\left((\mathbb{Z} / N \mathbb{Z})^{*}\right)$ will be Galois conjugates of each other. Since, $g$ is a minimal lift of $\bar{\rho}$, the $p$-part of $\epsilon\left((\mathbb{Z} / N \mathbb{Z})^{*}\right)$ is trivial. But the $p$-part of $\psi \epsilon\left((\mathbb{Z} / N \mathbb{Z})^{*}\right)$ is non-trivial as $\psi$ is a character of $p$-power order. So, they can't be Galois conjugates of each other. Therefore, $g$ and $h$ are not Galois conjugates of each other. Thus, $\mathfrak{m}$ is a congruence prime for $g$ and $H_{\emptyset}^{1}\left(G_{\mathbb{Q}}, A_{\rho_{g}}\right) \neq 0$. However, this does not ensure that $\amalg^{1}\left(G_{\mathbb{Q}, N p}, \omega_{p} \otimes \operatorname{ad}^{0} \bar{\rho}\right)$ is non-zero because we do not know whether the injection $\amalg^{1}\left(G_{\mathbb{Q}, N p}, \omega_{p} \otimes \operatorname{ad}^{0} \bar{\rho}\right) \rightarrow H_{\emptyset}^{1}\left(G_{\mathbb{Q}}, A_{\rho_{g}}\right)[\mathfrak{m}]$ given in the proof of Lemma 2.6 of [32], which we used in the last paragraph, is an isomorphism and in general, it is very difficult to prove such a result (see Lemma 2.6, remark 2.7 of [32] and the discussion before it for more details). But we do expect that $\amalg^{1}\left(G_{\mathbb{Q}, N p}, \omega_{p} \otimes \operatorname{ad}^{0} \bar{\rho}\right)$ is non-zero based on the calculations above.

Let us consider the modular representations coming from a weight 1 modular form. In the proposition below, we do not put the condition $p \mid \phi(N)$. For a number field $L$ with ring of integers $\mathcal{O}_{L}$, we denote by $A(L)[p]$ the $p$-torsion subgroup of the class group of $L$ and by $U(L)$ the kernel of the map $\frac{\mathcal{O}_{L}^{*}}{\left(\mathcal{O}_{L}^{*}\right)^{p}} \rightarrow\left(\prod_{v \mid p} \frac{\mathcal{O}_{L v}^{*}}{\left(\mathcal{O}_{L_{v}}^{*}\right)^{p}}\right)$.

Proposition 9. Let $\bar{\rho}$ be a $\Gamma_{1}(N)$-modular representation coming from a regular modular form $f$ of weight 1 which has either RM or CM by $F$ (see [7] for the definition of regular). Let $H$ be the extension of $\mathbb{Q}$ which is fixed by $\operatorname{Ker}\left(a d^{0} \bar{\rho}\right)$. Moreover, assume the following conditions:

(1) If $\ell \mid N$ and $p \mid \ell+1$, then $\ell$ does not stay inert in $F$.

(2) If $\ell$ is a prime divisor of $N$, then $\left.\bar{\rho}\right|_{G_{Q_{\ell}}}$ is irreducible.

(3) $A(F)[p]=0$ and $\operatorname{Hom}_{G}\left(A(H)[p], a d^{0} \bar{\rho}\right)=0$

(4) $\operatorname{Hom}_{G}\left(U(F), a d^{0} \bar{\rho}\right)=0$ and if $F$ is imaginary, then $\operatorname{Hom}_{G}\left(U(H), a d^{0} \bar{\rho}\right)=0$.

Then, $\bar{\rho}$ is unobstructed.

Proof. Note that, $\bar{\rho}=\operatorname{Ind}_{G_{F}}^{G_{\mathbb{Q}}} \delta$, where $F$ is a quadratic extension of $\mathbb{Q}$ which is either real or imaginary and $\delta: G_{F} \rightarrow \mathbb{F}^{*}$ is a character, as it comes from a weight 1 form which has $\mathrm{RM}$ or $\mathrm{CM}$. Let $\operatorname{Gal}(F / \mathbb{Q})=G^{\prime}=\{1, \sigma\}$. As $f$ is a regular weight 1 eigenform, $\left.\rho_{f}\right|_{G_{\mathbb{Q}_{p}}}=\chi_{1} \oplus \chi_{2}$, where $\chi_{1}$ and $\chi_{2}$ are distinct, unramified characters from $G_{\mathbb{Q}_{p}} \rightarrow\left(\overline{\mathbb{Q}_{p}}\right)^{*}$. 
So, $F$ and the fixed field $H$ of $\operatorname{Ker}\left(\operatorname{ad}^{0} \bar{\rho}\right)$ are unramified at $p$. From the hypothesis (2) above, we see that all prime divisors $\ell$ of $N$ are either inert or ramified in $F$.

Thus, from above, it follows that $\operatorname{ad}^{0} \bar{\rho}=\epsilon \oplus \operatorname{Ind}_{G_{F}}^{G_{\mathbb{Q}}} \chi$, where $\epsilon$ is the character of $G_{\mathbb{Q}}$ of order 2 corresponding to $F$ and $\chi=\delta / \delta^{\sigma}$. So, $H^{1}\left(G_{\mathbb{Q}, N p}, \operatorname{ad}^{0} \bar{\rho}\right)=H^{1}\left(G_{\mathbb{Q}, N p}, \epsilon\right) \oplus$ $H^{1}\left(G_{\mathbb{Q}, N p}, \operatorname{Ind}_{G_{F}}^{G_{\mathbb{Q}}} \chi\right)$. We will analyze each part of this sum separately. We shall mostly follow the notations of [7] below.

First, we will look at the group $H^{1}\left(G_{\mathbb{Q}, N p}, \epsilon\right)$ appearing above. From the inflationrestriction sequence, we see that $H^{1}\left(G_{\mathbb{Q}, N p}, \epsilon\right)=\left(\operatorname{Hom}\left(G_{F, S}, \mathbb{F}\right) \otimes \epsilon\right)^{G^{\prime}}$, where $S$ is the set of the places of $F$ dividing $N p$. From class field theory and the hypothesis (3) above, we have the following exact sequence of $G^{\prime}$-modules:

$$
0 \rightarrow \operatorname{Hom}\left(G_{F, S}^{\mathrm{ab}}, \mathbb{F}\right) \rightarrow \operatorname{Hom}\left(\prod_{v \mid N p} \mathcal{O}_{F_{v}}^{*}, \mathbb{F}\right) \rightarrow \operatorname{Hom}\left(\mathcal{O}_{F}^{*}, \mathbb{F}\right)
$$

Now, by hypothesis (1), we have:

$$
\operatorname{Hom}\left(\prod_{v \mid N p} \mathcal{O}_{F_{v}}^{*}, \mathbb{F}\right)=\operatorname{Hom}\left(\prod_{v \mid p} \mathcal{O}_{F_{v}} \prod_{\ell|N, p| \ell-1} \prod_{v \mid \ell} \mathbb{Z} /(\ell-1) \mathbb{Z}, \mathbb{F}\right)
$$

as $F$ is unramified at $p$. The factors in the second product come from places of $F$ dividing such primes and, since $\ell$ is either inert or ramified in $F$, each factor appears only once. Observe that $G^{\prime}$ acts trivially on each of them. Thus, $\left(\operatorname{Hom}\left(\prod_{\ell|N, p| \ell-1} \prod_{v \mid \ell} \mathbb{Z} /(\ell-\right.\right.$ 1) $\mathbb{Z}, \mathbb{F}) \otimes \epsilon)^{G^{\prime}}=0$.

If $F$ is imaginary, then $\mathcal{O}_{F}^{*}$ is a finite group and its order is not divisible by $p$, as $p$ is unramified in $F$. Thus, $U(F)=\mathcal{O}_{F}^{*} /\left(\mathcal{O}_{F}^{*}\right)^{p}=0$. Hence, in this case, it follows that $\left(\operatorname{Hom}\left(\prod_{v \mid p} \mathcal{O}_{F_{v}}, \mathbb{F}\right) \otimes \epsilon\right)^{G^{\prime}}$ has dimension 1. Therefore, $H^{1}\left(G_{\mathbb{Q}, N p}, \epsilon\right)$ has dimension 1. If $F$ is real, then the free part of $\mathcal{O}_{F}^{*}$ has rank 1 and the torsion part is $\{1,-1\}$. Suppose $p$ is not split in $F$. Then, it is inert in $F$ and the fundamental unit of $F$ generates the residue field of $\mathcal{O}_{F_{p}}$ over $\mathbb{F}_{p}$. Suppose $p$ is split in $F$. Then $\prod_{v \mid p} \mathcal{O}_{F_{v}}=\mathbb{Z}_{p} \times \mathbb{Z}_{p}$ and the action of $G^{\prime}$ switches them. Moreover, under the $G^{\prime}$-equivariant diagonal embedding $\mathcal{O}_{F}^{*} \rightarrow \prod_{v \mid p} \mathcal{O}_{F_{v}}^{*}=\mathbb{Z}_{p}^{*} \times \mathbb{Z}_{p}^{*}$, the fundamental unit of $F$ gets mapped to an element of the form $(a,-a)$ as the non-trivial element of $G^{\prime}$ sends the unit to its inverse. So, it follows, from the discussion above and hypothesis (4), that in both the cases, $G^{\prime}$ acts trivially on the subspace of elements of $\operatorname{Hom}\left(\prod_{v \mid p} \mathcal{O}_{F_{v}}, \mathbb{F}\right)$ which vanish on $\mathcal{O}_{F}^{*}$. An element of $\operatorname{Hom}\left(G_{F, S}^{\mathrm{ab}}, \mathbb{F}\right)$ vanishes on the fundamental unit. Thus, combining this and the previous paragraph, we see that $G^{\prime}$ acts trivially on $\operatorname{Hom}\left(G_{F, S}^{\mathrm{ab}}, \mathbb{F}\right)$, when $F$ is totally real. It follows that, in this case, $H^{1}\left(G_{\mathbb{Q}, N p}, \epsilon\right)=\left(\operatorname{Hom}\left(G_{F, S}^{\mathrm{ab}}, \mathbb{F}\right) \otimes \epsilon\right)^{G^{\prime}}=0$.

We shall now analyze the second factor appearing in the sum above. Let $H$ be the extension of $\mathbb{Q}$ which is fixed by $\operatorname{Ker}\left(\operatorname{ad}^{0} \bar{\rho}\right)$. Thus, $G=\operatorname{Gal}(H / \mathbb{Q})$ is a dihedral group $D_{2 n}$ which is non-abelian and $\operatorname{Ind}_{G_{F}}^{G_{\mathbb{Q}}} \chi$ is an irreducible representation of $G$. By the arguments 
used above, we get that $H^{1}\left(G_{\mathbb{Q}, N p}, \operatorname{Ind}_{G_{F}}^{G_{\mathbb{Q}}} \chi\right)=\left(H^{1}\left(G_{H, S}, \mathbb{F}\right) \otimes \operatorname{Ind}_{G_{F}}^{G_{\mathbb{Q}}} \chi\right)^{G}$. By hypothesis $(3), H^{1}\left(G_{H, S}, \mathbb{F}\right)$ is the subspace of $\operatorname{Hom}\left(\prod_{v \mid N p} \mathcal{O}_{H_{v}}^{*}, \mathbb{F}\right)$ vanishing on $\mathcal{O}_{H}^{*}$. Since $H$ is unramified at $p$, we get $\operatorname{Hom}\left(\prod_{v \mid N p} \mathcal{O}_{H_{v}}^{*}, \mathbb{F}\right)=\operatorname{Hom}\left(\prod_{v \mid p} \mathcal{O}_{H_{v}} \prod \mathbb{Z} / p \mathbb{Z}, \mathbb{F}\right)$ where the last product is taken over all places $v$ of $H$ which divide $N$ and whose residue fields have $p^{\text {th }}$ roots of unity. If $\ell$ is a such a prime, then the $G$-submodule $\operatorname{Hom}\left(\prod_{v \mid \ell} \mathbb{Z} / p \mathbb{Z}, \mathbb{F}\right)$ of the module above is isomorphic to $\operatorname{Ind}_{D_{\ell}}^{G} \alpha$, where $D_{\ell}$ is the image of $G_{\mathbb{Q}_{\ell}}$ under $\operatorname{ad}^{0} \bar{\rho}$ and $\alpha$ is the character by which it acts on the $p^{\text {th }}$ roots of unity. Note that, by hypothesis (2) above, $\operatorname{Ind}_{G_{F}}^{G_{\mathbb{Q}}} \chi$ is an irreducible representation of $D_{\ell}$ for every $\ell \mid N$. So, $\left(\operatorname{Hom}\left(\prod \mathbb{Z} / p \mathbb{Z}, \mathbb{F}\right) \otimes\right.$ $\left.\operatorname{Ind}_{G_{F}}^{G_{\mathbb{Q}}} \chi\right)^{G}=0$.

Assume $F$ is imaginary. As $H$ is unramified at $p$ and $p \nmid|G|$, we see, from using the arguments from [7, Section 3.2], that every irreducible representation of $G$ occurs in $\operatorname{Hom}\left(G_{H, S}^{\mathrm{ab}}, \mathbb{F}\right)$ with multiplicity at least 1 . The additional multiplicities would only arise from $U(H)$. But, from hypothesis (4) above, we have $\operatorname{Hom}_{G}\left(U(H), \operatorname{Ind}_{G_{F}}^{G_{\mathbb{Q}}} \chi\right)=0$. Therefore, $\operatorname{Ind}_{G_{F}}^{G_{\mathbb{Q}}} \chi$ occurs in $\operatorname{Hom}\left(G_{H, S}^{\mathrm{ab}}, \mathbb{F}\right)$ with multiplicity 1. Hence, $\left(\operatorname{Hom}\left(G_{H, S}, \mathbb{F}\right) \otimes\right.$ $\left.\operatorname{Ind}_{G_{F}}^{G_{\mathbb{Q}}} \chi\right)^{G}=H^{1}\left(G_{\mathbb{Q}, N p}, \operatorname{Ind}_{G_{F}}^{G_{\mathbb{Q}}} \chi\right)$ has dimension 1. If $F$ is real, then $\operatorname{Ind}_{G_{F}}^{G_{\mathbb{Q}}} \chi$ is totally odd. Thus, we see, from the arguments in [7, Section 3.2], that $\operatorname{Ind}_{G_{F}}^{G_{\mathbb{Q}}} \chi$ occurs in the subspace of $\operatorname{Hom}\left(\prod_{v \mid p} \mathcal{O}_{H_{v}}^{*}, \mathbb{F}\right)$ vanishing on $\mathcal{O}_{H}^{*}$ with multiplicity 2 . Note that, we do not need to consider contribution from $U(H)$ as $\operatorname{Ind}_{G_{F}}^{G_{\mathbb{Q}}} \chi$ does not occur in $\mathcal{O}_{H}^{*} /\left(\mathcal{O}_{H}^{*}\right)^{p}$. Therefore, combining this with the previous paragraph, we see that $\operatorname{Ind}_{G_{F}}^{G_{\mathbb{Q}}} \chi$ occurs in $\operatorname{Hom}\left(G_{H, S}^{\mathrm{ab}}, \mathbb{F}\right)$ with multiplicity 2 . Hence, $H^{1}\left(G_{\mathbb{Q}, N p}, \operatorname{Ind}_{G_{F}}^{G_{\mathbb{Q}}} \chi\right)$ has dimension 2.

Combining all the results above, we see that if $\bar{\rho}$ satisfies all the conditions above, then $H^{1}\left(G_{\mathbb{Q}, N p}, \operatorname{ad}^{0} \bar{\rho}\right)$ has dimension 2 . Hence, $\bar{\rho}$ is unobstructed in our sense.

Remark. (1) The hypotheses (3) and (4) are similar to those used by Mazur in sections 1.12 and 1.13 of [26] where he studies unobstructed representations unramified outside a single prime. Note that, for a number field $L, A\left(L\left(\zeta_{p}\right)\right)[p]=0$ implies that $U(L)=0$ (see remark after Proposition 1 of [10]).

(2) We know that $G^{\prime}=\operatorname{Gal}(F / \mathbb{Q})$ acts on $A(F)[p]$ by the character $\epsilon$. So, if $A(F)[p] \neq$ 0 , then it would contribute to $H^{1}\left(G_{\mathbb{Q}, N p}, \epsilon\right)$ in addition to what we have calculated above. Hence, in this case, we have $\operatorname{dim} H^{1}\left(G_{\mathbb{Q}, N p}, \epsilon\right)>1$. Thus, combining this with the other calculations above, we see that $\operatorname{dim} H^{1}\left(G_{\mathbb{Q}, N p}, \operatorname{ad}^{0} \bar{\rho}\right)>2$. Hence, if $A(F)[p] \neq 0$, then $\bar{\rho}$ will be obstructed.

(3) Suppose $f$ is a weight 1 form which has RM or CM by $F$, and there exists a prime $\ell$ such that $\ell \mid N$ and $p \mid \ell+1$. Moreover, assume that $\ell$ stays inert in $F$. Thus, using the notations of the proof above, we see that $\operatorname{Hom}\left(\prod_{v \mid N p} \mathcal{O}_{F_{v}}^{*}, \mathbb{F}\right)=$ $\operatorname{Hom}\left(\prod_{v \mid p} \mathcal{O}_{F_{v}} \prod_{\ell|N, p| \ell-1} \prod_{v \mid \ell} \mathbb{Z} /(\ell-1) \mathbb{Z} \prod \mathbb{Z} / p \mathbb{Z}, \mathbb{F}\right)$, where the last product is taken over all the prime divisors $\ell$ of $N$ which stay inert in $F$ and which are 
$-1 \bmod p$. Observe that, they are isomorphic to $\epsilon$ as $G^{\prime}$ representation. The projection of the image of $\mathcal{O}_{F}^{*}$ in $\prod_{v \mid N p} \mathcal{O}_{F_{v}}^{*}$ onto this product is also trivial as $F$ is unramified at $p$. Hence, this product also contributes to $H^{1}\left(G_{\mathbb{Q}, N p}, \epsilon\right)$, making it bigger. All the other calculations at the places above $p$ in the proof above still remain valid. As a result, we see that $\operatorname{dim} H^{1}\left(G_{\mathbb{Q}, N p}, \operatorname{ad}^{0} \bar{\rho}\right)>2$. Hence, in this case, $\bar{\rho}$ is obstructed.

Note that, the assumptions in the proposition above do not yield any immediate contradiction. Thus, we see that our notion of unobstructedness is weaker than Mazur's notion of unobstructedness.

Proposition 10. Let $\bar{\rho}$ be a modular representation satisfying the hypotheses of Proposition 9 and suppose $p \mid \phi(N)$. Let the $p$-primary part of $(\mathbb{Z} / N \mathbb{Z})^{*}$ be $\mathbb{Z} / p^{e_{1}} \mathbb{Z} \times \cdots \times \mathbb{Z} / p^{e_{n}} \mathbb{Z}$. Assume moreover that:

(1) $\bar{\rho}$ is a new $\Gamma_{1}(N)$-modular representation,

(2) For all primes $\ell$ dividing $N$ such that $p \mid \ell^{2}-1, U_{\ell}$ acts like 0 on all newforms of level $N$ which lift $\bar{\rho}$

Then, the corresponding local component $A_{\bar{\rho}}$ of the mod $p$ Hecke algebra is isomorphic to $\mathbb{F}[[x, y]]\left[y_{1}, \ldots, y_{n}\right] /\left(y_{1}^{p^{e_{1}}}, \ldots, y_{n}^{p^{e_{n}}}\right)$ and thus, is not reduced.

Proof. As $\bar{\rho}$ satisfies the hypotheses of Proposition 9, it satisfies the hypotheses of Theorem 3 as well. Indeed, $\bar{\rho}$ comes from a regular, weight 1 form and is unramified at $p$. So, $\left.\bar{\rho}\right|_{G_{\mathbb{Q}_{p}}}$ is of the required form. If $\ell$ is a prime divisor of $N$ which is $1 \bmod p$, then it splits completely in $\mathbb{Q}\left(\zeta_{p}\right)$. As $\left.\bar{\rho}\right|_{G_{\mathbb{Q}_{\ell}}}$ is irreducible, $\left.\bar{\rho}\right|_{G_{\mathbb{Q}(\zeta p)}}$ is also irreducible. Therefore, from the results of Böckle, Diamond, Diamond-Flach-Guo and Kisin, it follows that the surjective map $R_{\bar{\rho}} \rightarrow \mathbb{T}_{\bar{\rho}}^{\Gamma_{1}(N)}$ is an isomorphism. The hypothesis (2) along with the proof of Proposition 6 and the proof of Theorem 1 implies that the kernel of the surjective map $\mathbb{T}_{\bar{\rho}}^{\Gamma_{1}(N)} \rightarrow A_{\bar{\rho}}^{\Gamma_{1}(N)}$ is $m_{\Lambda} \mathbb{T}_{\bar{\rho}}^{\Gamma_{1}(N)}$. By Proposition 9, we see that $R_{\bar{\rho}} \simeq \mathcal{O}[[x, y, T]]\left[z_{1}, \cdots, z_{n}\right] /\left(z_{1}^{p^{e_{1}}}-1, \cdots, z_{n}^{p^{e_{n}}}-1\right)$. Combining all of the above gives us that $A_{\bar{\rho}} \simeq \mathbb{F}[[x, y]]\left[y_{1}, \cdots, y_{n}\right] /\left(y_{1}^{p^{e_{1}}}, \cdots, y_{n}^{p^{e_{n}}}\right)$ and hence, the proposition is proved.

Remark. (1) If the first condition of Proposition 10 is satisfied, then to check the second condition, it is sufficient, by Lemma 7 , to check that $\left.\bar{\rho}\right|_{I_{\ell}}$ is irreducible.

(2) Suppose $\bar{\rho}$ is a $\Gamma_{1}(N)$-modular representation which satisfies the two assumptions of Proposition 10 and the assumptions of Theorem 3. Moreover, assume $p \mid \phi(N)$ and let the $p$-primary part of $(\mathbb{Z} / N \mathbb{Z})^{*}$ be $\mathbb{Z} / p^{e_{1}} \mathbb{Z} \times \cdots \times \mathbb{Z} / p^{e_{n}} \mathbb{Z}$. Then, from the proof above, we see that $A_{\bar{\rho}}^{\Gamma_{1}(N)} \simeq R_{\bar{\rho}} /(\pi, T)$. Now, as $p \neq 2, R_{\bar{\rho}} \simeq$ $R_{\bar{\rho}}^{0}\left[T, y_{1}, \ldots, y_{n}\right] /\left(y_{1}^{p^{e_{1}}}-1, \ldots, y_{n}^{p^{e_{n}}}-1\right)$, where $R_{\bar{\rho}}^{0}$ is the universal deformation ring 
of $\bar{\rho}$ with constant determinant (see the proof of Lemma 19 of [8] for more details). So, $A_{\bar{\rho}}^{\Gamma_{1}(N)}$ is isomorphic to $\tilde{R}_{\bar{\rho}}^{0}\left[z_{1}, \ldots, z_{n}\right] /\left(z_{1}^{p^{e_{1}}}, \ldots, z_{n}^{p^{e_{n}}}\right)$ and hence, is not reduced. So, the assumptions of Proposition 9 are not necessary to get non-reduced Hecke algebras but are necessary to find the precise structure of the Hecke algebra.

We will now give some examples of non-reduced Hecke algebras following the previous remark. Let $\ell$ be a prime such that $p \mid \ell-1$ and $\ell$ is 3 modulo 4 . Let $K=\mathbb{Q}(\sqrt{-\ell})$ and $h_{K}$ be its class number. If $p$ splits in $K$, then define $n$ to be the smallest integer such that $p$ is not split in the anti-cyclotomic extension of degree $\ell^{n}$ of $K$ and $\ell^{n} \nmid h_{K}$. Otherwise, define it to be the smallest integer such that $\ell^{n} \nmid h_{K}$. Let $\chi: G_{K} \rightarrow \mathbb{Z}_{\ell} \rightarrow \mathbb{Z} / \ell^{n} \mathbb{Z} \rightarrow \overline{\mathbb{F}}^{*}$ where the first map is given by the anti-cyclotomic $\mathbb{Z}_{\ell}$ extension of $K$, and the last map is the inclusion of $\left(\ell^{n}\right)^{\text {th }}$ roots of unity into $\overline{\mathbb{F}}^{*}$. Now, we see, from a classical theorem of Hecke, that $\bar{\rho}=\operatorname{Ind}_{G_{K}}^{G_{\mathbb{Q}}} \chi$ of $G_{\mathbb{Q}, p \ell}$ is an odd, irreducible representation coming from a weight 1 newform $f$ of level $\ell^{2 n+3}$.

As $\chi^{\sigma}=\chi^{-1}$ and $\chi$ has odd order, they are distinct characters of $G_{K}$ and they only coincide on $\operatorname{ker}(\chi)$. Now, $\ell$ ramifies in $K$ and the prime $v^{\prime}$ of $K$ lying above $\ell$ ramifies in the anti-cyclotomic extension of $K$ of degree $\ell^{n}$ as $\ell^{n} \nmid h_{K}$. As a consequence, we see that $\left.\bar{\rho}\right|_{I_{\ell}}$ and $\left.\bar{\rho}\right|_{G_{\mathbb{Q}_{\ell}}}$ are irreducible. As $\ell$ splits completely in $\mathbb{Q}\left(\zeta_{p}\right)$, it follows that $\left.\bar{\rho}\right|_{G_{\mathbb{Q}\left(\zeta_{p}\right)}}$ is also irreducible. If $p$ is inert in $K$, then clearly $\left.\bar{\rho}\right|_{G_{\mathbb{Q}_{p}}}$ is a sum of distinct characters as $p$ is unramified in $K$. If $p$ is split in $K$, then for a place $v$ of $K$ above $p, \chi$ and $\chi^{\sigma}$ are distinct characters of $G_{K_{v}}$ as $\chi$ has odd order and $p$ does not split completely in the anti-cyclotomic extension of $K$ of degree $\ell^{n}$. As the anti-cyclotomic extension of $K$ is unramified at $p$, we see that $\left.\bar{\rho}\right|_{G_{\mathbb{Q}_{p}}}$ is not a direct sum of characters which are cyclotomic twists of each other. Therefore, $\bar{\rho}$ is a new $\Gamma_{1}\left(\ell^{2 n+3}\right)$-modular representation satisfying the two conditions of Proposition 10 and the conditions of Theorem 3. Hence, from the remark above, it follows that $A_{\bar{\rho}}^{\Gamma_{1}\left(\ell^{2 n+3}\right)}$ is not reduced.

\section{REFERENCES}

[1] F. Andreatta and E. Goren, Hilbert modular forms: $\bmod p$ and $p$-adic aspects, Mem. Amer. Math. Soc. 173 (2005), no. 819, vi+100 pp.

[2] A.O.L. Atkin and J. Lehner, Hecke operators on $\Gamma_{0}(m)$, Math. Ann., 185:134-160, 1970.

[3] J. Bellaïche, Une représentation galoisienne universelle attachée aux formes modulaires modulo 2, C.R.A.S. 350 (2012), no. 9-10, 443-448.

[4] J. Bellaïche, Pseudodeformations, Mathematische Zeitschrift 270 (2012), no. 3-4, 1163-1180.

[5] J. Bellaïche, Eigenvarieties, families of Galois representations, p-adic L-function, Course notes of a course given at Brandeis University in Fall, 2010, Available at http://people.brandeis.edu/ jbellaic/preprint/coursebook.pdf

[6] J. Bellaïche and G. Chenevier, Families of Galois representations and Selmer groups, Asterisque 324 (2009), pp. xii+314.

[7] J. Bellaïche and M. Dimitrov, On the eigencurve at classical weight one points, Duke Math. Journal, 165. 2 (2016), 245-266.

[8] J. Bellaïche and C. Khare, Level 1 Hecke algebras of modular forms modulo p, Compositio Mathematica 151. 3 (2015), 397-415. 
[9] G. Böckle, On the density of modular points in universal deformation spaces, Amer. J. Math. 123 (2001), no. 5, 985-1007.

[10] N. Boston, Deformations of Galois representations associated to the cusp form $\Delta$, Séminaire de Théorie des Nombres, Paris 1987-88, 51-63.

[11] H. Carayol, Sur les représentations galoisienne modulo $\ell$ attachées aux formes modulaires, Duke Math. Journal 59 (1989), 785-801.

[12] G. Chenevier, The p-adic analytic space of pseudocharacters of a profinite group and pseudorepresentations over arbitrary rings, Proceedings of the LMS Durham Symposium, Automorphic Forms and Galois Representations (2011).

[13] G. Chenevier, On the infinite fern of Galois representations of unitary type, Annales Scientifiques de l'E.N.S. 44 (2011), 963-1019.

[14] F. Diamond, On deformation rings and Hecke rings, Annals of Math. 144 (1996), 137-166.

[15] F. Diamond, M. Flach and L. Guo, The Tamagawa number conjecture of adjoint motives of modular forms, Ann. Sci. Ec. Norm. Sup. 37 (2004), 663-727.

[16] D. Eisenbud, Commutative algebra with a view toward algebraic geometry, Graduate Texts in Mathematics, 150, Springer-Verlag, New York, 1995.

[17] M. Emerton, p-adic families of modular forms (after Hida, Coleman and Mazur), Séminaire Bourbaki, Vol. 2009/2010, Exposés 1012-1026, Astérisque No. 339(2011), Exp. no. 1012, vii, 31-61.

[18] M. Emerton, R. Pollack, and T. Weston, Variation of Iwasawa invariants in Hida families, Invent. Math., 163(3): 523-580, 2006.

[19] F. Gouvêa, Arithmetic of p-adic modular forms, Lecture Notes in Mathematics, 1304, Springer Verlag, Berlin, 1988.

[20] F. Gouvêa and B. Mazur, On the density of modular representations, Computational perspectives on number theory (Chicago, IL, 1995), 127-142, AMS/IP Stud. Adv. Math., 7, Amer. Math. Soc., Providence, RI, 1988.

[21] H. Hida, Iwasawa modules attached to congruences of cusp forms, Annales de l'ENS, 4th series 19 (1986), 231-273

[22] N. Jochnowitz, A study of the local components of the Hecke algebra mod l, Trans. Amer. Math. Soc. 270 (1982), no. 1, 253-267.

[23] N. Katz, Higher congruences between modular forms, Ann. Math. (2) 101 (1975), 332-367.

[24] C. Khare, Mod p modular forms, Number theory (Tiruchirapalli, 1996), Contemp. Math., 210, Amer. Math. Soc., Providence, RI, 1998, 135-149.

[25] M. Kisin, Geometric deformations of modular Galois representations, Invent. Math. 157 (2004), no. 2, 275-328.

[26] B. Mazur, Deforming Galois representations, Galois groups over $\mathbb{Q}$ (Berkeley, CA, 1987), 385-437, Math. Sci. Res. Inst. Publ., 16, Springer, New York, 1989.

[27] J-L. Nicolas and J-P. Serre, L'ordre de nilpotence des opérateurs de Hecke modulo 2, C.R.A.S. 350 (2012), no. 7-8, 343-348.

[28] J-L. Nicolas and J-P. Serre, Formes modulaires modulo 2 : structure de l'algébre de Hecke, C.R.A.S. 350 (2012), no. 9-10, 449-454.

[29] R. Rouquier, Caractérisation des caractéres et pseudo-caractéres, J. Algebra 180(2) (1996), 571-586.

[30] L. Washington, Galois Cohomology, Modular forms and Fermat's last theorem (Boston, MA, 1995), 101-120, Springer, New York, 1997.

[31] T. Weston, Unobstructed modular deformation problems, American Journal of Mathematics 126(2004), 1237-1252.

[32] T. Weston, Explicit unobstructed primes for modular deformation problems of squarefree level, Journal of Number Theory (special issue in honor of Arnold Epharim Ross) 110 (2005), 199-218.

E-mail address: deoshaunak@gmail.com

Department of Mathematics, MS 050, Brandeis University, 415 South Street, Waltham, MA 02453, USA

Current address: Université du Luxembourg, Faculté des Sciences, de la Technologie et de la Communication, 6, rue Richard Coudenhove-Kalergi, L-1359 Luxembourg, Luxembourg 